\title{
28 Research Square \\ Batch Extraction of Tree Stem From Natural Forest Based on Terrestrial Laser Scanning
}

Jianpeng Zhang

Yunnan Normal University

Jinliang Wang ( $\nabla_{\text {jlwang@ynnu.edu.cn ) }}$

Yunnan Normal University https://orcid.org/0000-0001-7202-646X

Weifeng Ma

Yunnan Normal University

Yicheng Liu

Yunnan Normal University

Qianwei Liu

Yunnan Normal University

Zhiyan Zhang

Yunnan Normal University

\section{Research}

Keywords: Density clustering, Natural forest, Point cloud feature, Shangri-La, Tree stem, Terrestrial laser scanning

Posted Date: November 19th, 2020

DOI: https://doi.org/10.21203/rs.3.rs-108535/v1

License: (c) (i) This work is licensed under a Creative Commons Attribution 4.0 International License.

Read Full License 


\section{Abstract}

Background: Aiming at the problems of low accuracy of tree stem extraction from point cloud data of natural forest and poor universality, a method for batch extraction of tree stem from natural forest point cloud data based on terrestrial laser scanning is proposed.

Methods: First, the principal component analysis method is used to calculate the point cloud eigenvalues and eigenvectors, and the information entropy is minimized as a constraint to achieve the best neighborhood scale selection; Then combined with the spatial distribution features of the threedimensional forest, using the Z-axis component of normal vector as the feature variable, the threshold method is used to filter out a large number of non-stem point clouds, and the 3D features are used for rough extraction of tree stem point cloud; Finally, density clustering is used to realize the precise extraction of tree stem point cloud.

Results: Select the two typical representative natural forest sample plots of Pinus densata Mast. and Picea asperata Mast. in Shangri-La as the experimental data to extract stem. All the stem of the two natural forest sample plots were detected and extracted. Using the extracted individual tree stem point cloud and the true tree stem point cloud for correlation analysis, the $\mathrm{R}^{2}$ of the Pinus densata Mast. sample plot was 0.990 , and the $\mathrm{R}^{2}$ of the Picea asperata Mast. sample plot with a more complex growth environment was 0.982 .

Conclusions: The results show that this method can well achieve batch extraction of tree stem point cloud from natural forest, and has the characteristics of high extraction accuracy and strong adaptability.

\section{Background}

The stem of tree bears main function of nutrient transportation and is an important part of the forest (Leopold 1971). The growth morphology of tree stem is one of the important indicators for evaluating forests (Maltamo et al. 2006; Chiba 2000; Laasasenaho et al. 2005; Yu et al. 2013). Meanwhile, The measurement and statistics of tree parameters such as tree height and diameter at breast height (DBH) often start from the tree stem (Li et al. 2012; Liu et al. 2016; Liu et al. 2018), so the tree stem has important economic and Ecological value. The traditional forest tree stem survey method requires researchers to go deep into the forest area and use relevant instruments to perform measurement statistics (Meng 2006). Although this method can ensure the measurement accuracy, the main disadvantage of this method is time-consuming, laborious, and inefficient. The emergence and development of Light Detection And Ranging (LiDAR) remote sensing technology provides a new method for forest ecological investigation and research (Li et al. 2016; Pang et al. 2019; Liu et al. 2017; Yan et al. 2018). It can efficiently and accurately obtain the three-dimensional spatial structure of the forest, and further obtain forest information, which makes up for the shortcomings of traditional methods. Airborn Laser Scanning (ALS) and Terrestrial Laser Scanning (TLS) are the two main LiDAR technologies currently used in forest application research (Liu and Pang 2014). Because ALS technology is difficult to 
obtain structural information below the forest canopy, it is mainly used in researches such as forest biomass and canopy parameter inversion (Li et al. 2015; Li et al. 2015; Xie et al. 2020). Compared with the ALS technology, the point cloud data obtained by the TLS technology can clearly and accurately characterize the three-dimensional spatial structure below the forest canopy, and has a great advantage in the research under the forest canopy. Therefore, the current research related to tree stem is mostly based on TLS technology (Tao et al. 2015; Ma et al. 2019; Wang 2019).

At present, many scholars have carried out many studies on the detection and extraction of tree stem using TLS technology. Common research methods can be roughly divided into two categories. One method is to perform cylindrical fitting for tree stem point cloud. For example, Olofsson et al. (2014) performed cylindrical fitting for tree stem point cloud based on RANSAC algorithm to obtain the trunk model. Duan and Liu (2020) processed point cloud data for filtering, smoothing and repair firstly, and then carried out modeling and extraction for tree stem point cloud based on PROSAC algorithm. Liang et al. (2012) used the local normal vector feature of the point cloud to extract the tree stem roughly, and then performed cylindrical fitting on the tree stem point cloud to obtain its growth model. The stem growth model constructed by this kind of method can directly represent the stem information, but its accuracy is easily affected by the growth environment, growth morphology and point cloud quality of the forest. Another method is to use the local neighborhood features of the point cloud to extract the tree stem point cloud. For example, Xia et al. (2015) extracted bamboo stem based on the linear features of the local neighborhood of the point cloud. Luo et al. (2019) extracted the stem based on the consistency of the main direction of the stem point cloud. Liang et al. (2014) extracted tree stem by comparing the discreteness of local point clouds, and believed that the smaller the discreteness of local point clouds, the greater the probability of the stem points. This kind of method can detect and extract forest tree stem point cloud to a certain extent, but it is still challenging to extract the high-precision point cloud of tree stem in natural forest using only a single point cloud feature. For example, the linear feature of the point cloud is not sensitive to the stem of trees with large DBH in natural forest.

In summary, in order to solve the problems of low accuracy and low universality of tree stem extraction, this paper proposes a method for batch extraction of tree stem from natural forest based on TLS point cloud data. The study uses the information entropy minimization as the constraint condition to adaptively obtain the best neighborhood radius to calculate the Z-axis component of the point cloud normal vector and the 3D features, combined with the Density-Based Spatial Clustering of Applications with Noise (DBSCAN) algorithm to achieve High-precision extraction of point clouds of tree stem from natural forest.

\section{Materials And Methods}

\section{Study area and data acquisition}

Shangri-La City is located in the northwestern part of Yunnan Province, China (Fig. 1). It has rich vegetation types and extensive natural forest coverage (Yu et al. 2019; Chen et al. 2019). The main forest 
types include Pinus yunnanensis Franch, Pinus densata Mast., and Picea asperata Mast. et al. Two typical and representative Pinus densata Mast. and Picea asperata Mast. forest sample plots were selected as experimental data in the study area. The center coordinates of the Pinus densata Mast. natural forest sample plot is $\mathrm{N} 27^{\circ} 38^{\prime}, \mathrm{E} 99^{\circ} 45^{\prime}$, and the elevation is $3269 \mathrm{~m}$. There are few shrubs and weeds under the forest, and the trees grow sparsely. The center coordinates of the Picea asperata Mast. natural forest sample plot is $\mathrm{N} 27^{\circ} 48^{\prime}, \mathrm{E} 99^{\circ} 59^{\prime}$, and the elevation is $3697 \mathrm{~m}$. The growth environment under the forest is complex, with bushes and weeds growing, and trees growing densely. The study uses Leica P40 3D laser scanner as the data acquisition device. The Leica P40 3D laser scanner is an excellent TLS point cloud data acquisition device that can efficiently and accurately acquire high-density point clouds. Its performance parameters are shown in Table 1. In order to obtain the point cloud data of the forest sample plot more completely, 3 inter-visibility targets were placed in the forest and scanned at 5 sites ( 1 center in the center and 4 nearby). After obtaining the cloud data of each site in the forest sample plot, the original point cloud data was obtained by stitching them according to the target position in the cyclone software, and finally the experimental data was obtained by denoising and cropping(Fig. 2).

Table 1

Leica P40 LiDAR specifications

\begin{tabular}{|ll|}
\hline Indicator & Description \\
\hline Range accuracy & $1.2 \mathrm{~mm}+10 \mathrm{ppm}$ \\
\hline 3D position accuracy & $\begin{array}{l}3 \mathrm{~mm} @ 50 \mathrm{~m} \\
6 \mathrm{~mm} @ 100 \mathrm{~m}\end{array}$ \\
\hline Wavelength & $1,550 \mathrm{~nm}$ (invisible); $658 \mathrm{~nm}$ (visible) \\
\hline Scan rate & Up to 1,000,000 points per second \\
\hline Field of view & 360 ॰ (horizontal); 290。 (vertical) \\
\hline Range and reflectivity & Minimum range: $0.4 \mathrm{~m}$ \\
& Maximum range at reflectivity: \\
& $120 \mathrm{~m}$ (8\%), $180 \mathrm{~m}$ (18\%), 270 m (34\%) \\
& $0.4 \mathrm{~mm}$ RMS at $10 \mathrm{~m}$ \\
\hline Range noise & $0.5 \mathrm{~mm}$ RMS at $50 \mathrm{~m}$ \\
\hline
\end{tabular}

\section{Research process}

Different types of point clouds have different three-dimensional spatial geometric characteristics, and point cloud features have important applications in point cloud classification research (Yang and Dong 2013; Wang et al. 2019; Wang et al. 2019; Zhou et al. 2019; Zhao et al. 2020). There are two main characteristics of tree stem: (1) From an overall perspective, affected by the growth law of trees, the stem 
are distributed vertically upwards and grow upwards basically perpendicular to the ground. (2) In a local area, it is generally cylindrical, while other non-stem point clouds have basically no three-dimensional features. According to the above-mentioned spatial distribution characteristics of tree stem, a tree stem extraction method based on TLS natural forest point cloud data is proposed, which is divided into two steps: rough extraction and precise extraction. The first step: Firstly,calculate the normal vector feature of the local neighborhood of the forest point cloud, and find its component $N_{Z}$ on the $Z$ axis. The smaller the $N_{Z}$, the greater the probability that it is a tree stem point (Liang et al. 2012). Then, set a reasonable $N_{Z}$ threshold to cycle and remove non-tree stem point clouds such as ground, shrubs and canopy to the greatest extent. Finally, according to the principle that the stem are distributed in a columnar shape in the local neighborhood, the forest tree stem are roughly extracted in combination with the 3D features. The second step: There are still some non-stem points around the rough tree stem point cloud extracted through the above steps. According to the feature that the tree stem point cloud is densely distributed in a certain xy plane, the distribution of non-tree stem points is relatively scattered. The DBSCAN algorithm is used to cluster the xy plane, and a small number of outliers are denoised and optimized to obtain a precise point cloud of the stem. The technical roadmap is shown in Fig. 3.

\section{Rough extraction of tree stem point cloud}

The point cloud normal vector feature and dimensional feature can be represented by the eigenvalues and eigenvectors of the covariance matrix of the point cloud within its local neighborhood radius $R$ (An et al. 2018). Calculate the eigenvalues $\left(\lambda_{1}, \lambda_{2}, \lambda_{3}, \lambda_{1}>\lambda_{2}>\lambda_{3}\right)$ and eigenvectors $\left(n_{1}, n_{2}, n_{3}\right)$ in the local neighborhood radius $R$ of the forest point cloud by using the Principal Component Analysis (PCA) algorithm (Hoppe et al. 1992). The eigenvector corresponding to the smallest eigenvalue is the normal vector. The calculation methods of the $N_{Z}$ (Liang et al. 2012) value and dimensional feature line $\left(\mathrm{a}_{1 \mathrm{D}}\right)$, plane $\left(a_{2 D}\right)$ and volume $\left(a_{3 D}\right)$ (Ma et al., 2020) of the normal vector of the point cloud are shown in formulas (1) and (2).

$$
N_{z}=\left|n_{3} \times n_{0}\right|
$$

where, $n_{0}$ represents the vector $[0,0,1]$.

$$
a_{1 \mathrm{D}}=\frac{\sqrt{\lambda_{1}}-\sqrt{\lambda_{2}}}{\sqrt{\lambda_{1}}}, a_{2 \mathrm{D}}=\frac{\sqrt{\lambda_{2}}-\sqrt{\lambda_{3}}}{\sqrt{\lambda_{1}}}, a_{\mathrm{iD}}=\frac{\sqrt{\lambda_{3}}}{\sqrt{\lambda_{1}}}
$$

The size of the local neighborhood radius $R$ of the point cloud has a greater impact on the features in the point cloud. The local features of a point cloud will change due to different neighborhood radius, so the use of a unified neighborhood radius will affect the extraction accuracy of the tree stem point cloud (Ma et al. 2019; Xuan et al. 2019). This paper takes the information entropy minimization as the constraint condition to adaptively obtain the best neighborhood radius $R$ to reduce the error (Demantké et al. 2011). The principle is shown in formula (3). 


$$
E_{p}=-a_{1 \mathrm{D}} \ln \left(a_{1 \mathrm{D}}\right)-a_{2 \mathrm{D}} \ln \left(a_{2 \mathrm{D}}\right)-a_{3 \mathrm{D}} \ln \left(a_{3 \mathrm{D}}\right)
$$

where, $E p$ represents the information entropy of point $p$ in a certain neighborhood radius. The smaller the $E p$ value, the more unitary the dimensional features of the point $p$ in the neighborhood, and the neighborhood radius is expressed as the best point $p$ radius. The steps for calculating the best neighborhood radius $R$ of point $\mathrm{p}$ are: Firstly, determine a neighborhood radius interval [Rmin, Rmax] and radius increment $\Delta R$. Then, loop to calculate the $E p$ value of all radius of point $p$ in this radius interval. Finally, the $E p$ value is compared, when the $E p$ value is the smallest, the corresponding radius $R$ is the optimal radius.

Obtain the eigenvalue and eigenvalue vector corresponding to the best neighborhood radius $R$ of the point, and calculate the normal vector Z-axis component $N_{Z}$ to filter out most of the non-tree stem points such as the ground, shrub and canopy. Then use the three-dimensional spatial characteristics of the remaining point cloud to further roughly extract the tree stem point cloud.

\section{Precise extraction of tree stem point cloud}

The DBSCAN algorithm is an algorithm based on density clustering. It does not need to know the number of clusters, can find clusters of any shape, and can also identify noise points (Ester et al. 1996). Suppose that the points $p, q$, and $c$ are three points in the point cloud set $D$. In the DBSCAN algorithm, several important definitions are as follows. (1) Parameters Eps and MinPts: They respectively represent the radius of the spherical neighborhood of a point and the minimum number of points forming a cluster. These two parameters need to be defined in advance. (2) Core points, edge points and outlier points: If the number of points in the Eps neighborhood of point $p$ is greater than MinPts, then point $p$ is called the core point. If the number of points in the Eps $\mathrm{n}$ eighborhood of point $q$ is less than MinPts, but point $q$ is in the Eps neighborhood of point $p$ or other core points, the point $q$ is called the boundary point. The point that does not meet the above two conditions is called the noise point. (3) Directly density-reachable, density-reachable and density-connected (Guo et al. 2018): if point $p$ is the core point, and point $q$ is in the Eps neighborhood of point $p$, then point $q$ is called the directly density-reachable of point $p$. If the point $q$ is directly density-reachable of point $p$, and the point $c$ is directly density-reachable of point $q$, then point $c$ is called the density-reachable of point $p$. But the density-reachable is only between the core points. If point $p$ and point $q$ are all density-reachable of point $c$, then point $p$ and point $q$ are called densityconnected. The principle of clustering in the DBSCAN algorithm is to find the density-connected maximum point set in the point cloud set $D$ to form a cluster, which achieve the purpose of clustering.

According to the principle of the DBSCAN algorithm, the tree stem are clustered and then extracted, and a small number of outliers are denoised and optimized to realize the precise extraction of the tree stem point cloud.

\section{Results}


Matlab R2019b was used as the experimental platform to process the cloud of the Pinus densata Mast. sample plot that total number of point is 1972282 . Set the neighborhood radius interval to [0.1, 1], the increment $\Delta R$ is 0.2 , calculate the best neighborhood radius of the point, and get the eigenvalue and eigenvector corresponding to the best neighborhood radius.

The first step is to roughly extract the stem points. Firstly, calculate the $N_{Z}$ value with the normal vector. Then set a reasonable $N_{Z}$ threshold $T$, and extract points with $N_{Z}<T$ to reduce the influence of non-stem points on the extraction of tree stem. When the threshold $T$ is 0.1 and 0.2 , the results of removing ground, shrub and canopy points are shown in Fig. 4 . It can be seen from Fig. 4 that when $T$ is 0.1 and 0.2 , the stem point will be missing (the red part in the Fig. 4) for the sloping and curved stem.

When the threshold $T$ is 0.3 and 0.4 , the results of removing ground, shrub and canopy points are shown in Fig. 5. It can be seen from Fig. 5 that the stem points of the sloping and curved tree can be preserved. However, when $T$ is 0.3 , the stem point cloud can be completely retained, while the ground point cloud, shrub point cloud and canopy point cloud can be filtered out to the maximum. Therefore, we loop this step with the extraction result of $T$ is 0.3 , filter out most of the non-stem point clouds, and minimize the influence of other types of point clouds on the extraction of tree stem point clouds. After 5 looped, we found that the non-stem points have been removed to the maximum extent, and the result is shown in Fig. 6.

Finally, 3D features are used for the remaining points to further remove the non-stem point cloud, and obtain the rough extraction result of the stem. In order to determine the size of the 3D features value of the point cloud of the tree stem, the tree stem within the height range of $3 m-6 m$ from the experimental data are sampled and the 3D features value of each point is counted. Figure 7(a) shows the numerical distribution of the tree stem point cloud in the 3D features, indicating that the point with the 3D features value roughly within [0.05-0.5] is the tree stem point. Extract the points whose 3D features values of the remaining points in the previous step are within [0.05-0.5], and get the rough extraction result of the tree stem point cloud, as shown in Fig. 7(b).

The second step is the precise extraction of the tree stem point cloud. The DBSCAN algorithm is more sensitive to two input parameters Eps and MinPts. When Eps is larger and MinPts is smaller, the number of clusters will decrease, causing other types of point clouds to be classified as tree stem point clouds. When Eps is smaller and MinPts is larger, the number of clusters will increase, resulting in tree stem point clouds being classified as other types of point clouds. After research and comparison, for the experimental point cloud of the Pinus densata Mast. sample plot, it is found that when Eps is in the interval of $[0.02,0.04]$ and MinPts is in the interval of $[20,30]$, the tree stem point cloud extraction can obtain higher accuracy results. Figure 8 shows the precise extraction results of the stem when Eps is 0.03 , MinPts is 25 , and a small amount of outlier noise points are removed.

In terms of the number of stem identified, the method in this paper can detect and extract all the stem in the Pinus densata Mast. sample plot with a precision of $100 \%$. In order to verify the accuracy of the 
extraction of each single tree stem, the tree stem of the original experimental data were manually extracted. Correlation analysis was performed between the stem extracted manually and the stem points extracted by the method in this paper, and $\mathrm{R}^{2}$ was 0.990 , as shown in Fig. 9 . It shows that the tree stem points extracted by this method have high accuracy.

The experiment was conducted on Picea asperata Mast. sample plot with a more complex growth environment and densely growing trees. The number of points was 2725298 . The results of the stem are shown in Fig. 10. The stem of the Picea asperata Mast. sample plot were manually extracted and the accuracy was analyzed. The results show that the method in this paper can still detect all the stem in the sample plot, and the extraction accuracy of the number of stem is $100 \%$. The same correlation analysis was performed between the stem extracted manually and the stem points extracted by the method in this paper, and $\mathrm{R}^{2}$ was 0.982 , as shown in Fig. 11 . The results show that in natural forests with complex growth environment, this method can still obtain high-precision stem extraction results.

\section{Discussion}

At the end, we analyzed the factors that affect the accuracy of stem extraction, and compared the stem extraction results of the two sample plots, as shown in Fig. 12. From the extraction results of the stem of the two sample plots, it can be seen that the stem below the canopy can be extracted with high precision, and the stem with branches can also be extracted (Pinus densata Mast..(e) in Fig. 12), the error mainly comes from the upper part of the canopy. We found that there are two main factors affecting the extraction of stem from the upper part of the canopy. (1) Differences in tree species growth. Compared with the Picea asperata Mast. sample plot, the upper part of the stem of the Pinus densata Mast. sample plot is not cleanly extracted, and there are some secondary branch point clouds. This is mainly related to the difference in the growth morphology of the two tree species. There are many secondary branches growing in the Pinus densata Mast. canopy, and the growth is relatively dense. Therefore, during the extraction of the stem, there are still many secondary branch point clouds on the upper part of the stem of the Pinus densata Mast. sample plot that are not easy to remove, which affects the extraction accuracy. (2) Canopy occlusion. From the extraction results of the two plots, the tree stem in the upper part of the canopy have missing point clouds. This is mainly because when data is collected, due to the growth of natural forest trees, the upper part of the canopy is severely occlusion from each other, and it is difficult to obtain a complete point cloud of the upper part of the canopy. Therefore, as the height of the tree increases, the stem extraction accuracy is also decreasing.

\section{Conclusions}

In order to solve the problems of low accuracy of tree stem extraction from natural forest and poor universality, the research proposed a method for batch extraction of tree stem based on TLS point cloud data of natural foerest. The Z-axis component of the normal vector and the 3D features of the point cloud are calculated through the adaptive neighborhood radius, and finally the DBSCAN algorithm is used to extract the stem of the natural forest. All the stem of the two natural forest sample plots were detected 
and extracted. Through correlation analysis with the manually extracted stem points, it was found that the stem extraction $\mathrm{R}^{2}$ of the Pinus densata Mast. sample plot was 0.990 , indicating that the extraction accuracy was high. High-precision stem extraction results were still obtained in Picea asperata Mast. sample plot with complex growth environment. Stem extraction $\mathrm{R}^{2}$ of Picea asperata Mast. sample plot was 0.982 . However, it is also found that the two factors of tree species growth difference and canopy occlusion have a greater impact on the extraction accuracy of the upper part of the stem. How to reduce the influence of these two factors on the extraction of the stem and further improve the extraction accuracy will be the future research focus.

\section{Abbreviations}

DBH: Diameter at breast height; LiDAR: Light Detection And Ranging; ALS: Airborn laser scanning; TLS: Terrestrial laser scanning; DBSCAN: Density-based spatial clustering of applications with noise

\section{Declarations}

\section{Acknowledgements}

Not applicable.

\section{Author' contributions}

Jinliang Wang, Weifeng Ma and Jianpeng Zhang conceived and designed the experiments; Jianpeng Zhang and Qianwei Liu performed the experiments; Yicheng Liu and Zhiyan Zhang analyzed the data; and Jianpeng Zhang wrote the paper. All authors read and approved the final manuscript.

\section{Funding}

This research was funded by the National Natural Science Foundation of China for the "Natural Forests Biomass Estimation at Tree Level in Northwest Yunnan by Combining ULS and TLS Cloud Points Data", grant number: 41961060. This research was funded by the Multi-government International Science and Technology Innovation Cooperation Key Project of National Key Research and Development Program of China for the"Environmental monitoring and assessment of land use / land cover change impact on ecological security using geospatial technologies" , grant number: 2018YFE0184300. This research was funded by the Program for Innovative Research Team (in Science and Technology) in the University of Yunnan Province, IRTSTYN.

\section{Availability of data and materials}

The datasets used and/or analyzed during the current study are available from the corresponding author on reasonable request.

\section{Ethics approval and consent to participate}


The subject has no ethic risk.

\section{Consent for publication}

Not applicable.

\section{Competing interests}

The authors declare that they have no competing interests

\section{References}

1. An YJ, Chen XX, Sui LC, Zhou RR (2018) A Structural Road Extraction Method Based on Normal Vectors Similarity of Point Clouds. Bulletin of Surveying and Mapping. 11: 69-72. https://doi.org/10.13474/j.cnki.11-2246.2018.0352.

2. Chen Y, Wang JL, Liu GJ, Yang YL, Liu ZY, Deng H (2019) Hyperspectral Estimation Model of Forest Soil Organic Matter in Northwest Yunnan Province, China. Forests, 10: 217. https://doi.org/10.3390/f10030217.

3. Chiba Y (2000) Modelling stem breakage caused by typhoons in plantation cryptomeria japonica forests. For. Ecol. Manage. 135: 123-131. https://doi.org/10.1016/S0378-1127(00)00304-2.

4. Demantké J, Mallet C, David N, Vallet B (2011) Dimensionality based scale selection in 3D lidar point clouds. ISPRS - International Archives of the Photogrammetry Remote Sensing and Spatial Information Sciences, 38: 97-102. https://doi.org/10.5194/isprsarchives-XXXVIII-5-W12-97-2011.

5. Duan XJ, Liu MS (2020) Tree Trunk Extraction of the Stand Based on TLS of PROSAC Algorithm. Journal of Northwest Forestry University. 35: 226-231+256. https://doi.org/10.3969/j.issn.10017461.2020.01.34.

6. Ester M, Kriegel H, Sander J, Xu, X (1996) A Density-Based Algorithm for Discovering Clusters in Large Spatial Databases with Noise. In: Proceedings of $2^{\text {nd }}$ International Conference on Knowledge Discovery and Data Mining. 96: 6-231.

7. Guo QH, Su YJ, Hu TY, Liu J (2018) LiDAR principles, processing and applications in forest ecology. Higher Education Press: Beijing. pp. 128-129.

8. Hoppe H, DeRose T, Duchamp T, McDonald J, Stuetzle W (1992) Surface reconstruction from unorganized points. ACM SIGGRAPH Computer Graphics. 26: 71-78.

https://doi.org/10.1145/133994.134011.

9. Laasasenaho J, Melkas T, Aldén S (2005) Modelling bark thickness of picea abies with taper curves. For. Ecol. Manage. 206: 35-47. https://doi.org/10.1016/j.foreco.2004.10.058.

10. Leopold LB (1971) Trees and streams: the efficiency of branching patterns. J. theor. Biol. 31: 339354. https://doi.org/10.1016/0022-5193(71)90192-5.

11. Li D, Pang Y, Yue CR, Zhao D, Xu GC (2012) Extraction of individual tree DBH and height based on terrestrial laser scanner data. J. Beijing For. Univ. 34: 79-86. https://doi.org/10.13332/j.1000- 
1522.2012.04.027.

12. Li W, Niu Z, Huang N, Wang C, Gao S, Wu CY (2015) Airborne LiDAR technique for estimating biomass components of maize: A case study in Zhangye City, Northwest China. Ecol. Indic. 57: 486496. https://doi.org/10.1016/j.ecolind.2015.04.016.

13. Li WJ, Zhao CY, Bie Q, Gao CC, Gao YF (2015) Retrieval of the Forest Structural Parameters Using Airborne LiDAR Data. Remote Sens. Technol. Appl. 30: 917-924.

14. Li ZY, Liu QW, Pang Y (2016) Review on forest parameters inversion using LiDAR. J. Remote Sens. 20: $1138-1150$.

15. Liang XL, Kankare V, Yu XW, Hyyppa J, Holopainen M (2014) Automated Stem Curve Measurement Using Terrestrial Laser Scanning. IEEE Trans. Geosci. Remote Sens.52: 1739-1748. https://doi.org/10.1109/tgrs.2013.2253783.

16. Liang XL, Litkey P, Hyyppa J, Kaartinen H, Vastaranta M, Holopainen M (2012) Automatic Stem Mapping Using Single-Scan Terrestrial Laser Scanning. IEEE Trans. Geosci. Remote Sens. 50: 661670. https://doi.org/10.1109/tgrs.2011.2161613.

17. Liu GJ, Wang JL, Dong PL, Chen Y, Liu ZY (2018) Estimating individual tree height and diameter at breast height (DBH) from terrestrial laser scanning (TLS) data at plot level. Forests. 9: 398. https://doi.org/10.3390/f9070398.

18. Liu LX, Pang Y (2014) Applications of Airborne Laser Scanning and Terrestrial Laser Scanning to Forestry. World Forestry Research. 27: 49-56. https://doi.org/10.13348/j.cnki.sjlyyj.2014.01.009.

19. Liu LX, Pang Y, Li ZY (2016) Individual Tree DBH and Height Estimation Using Terrestrial Laser Scanning (TLS) in A Subtropical Forest. Sci. Silvae Sin. 52: 26-37.

20. Liu QW, Li SM, Li ZY, Fu LY, Hu KL (2017) Review on the Applications of UAV-Based Li DAR and Photogrammetry in Forestry. Scientia Silvae Sinicae., 53: 134-148. https://doi.org/10.11707/j.10017488.20170714.

21. Luo YB, Huang HY, Tang LY, Chen CC, Zhang H (2019) Tree Height and Diameter Extraction with 3D Reconstruction in a Forest based on TLS. Remote Sens. Technol. Appl. 34: 243-252.

22. Ma WF, Wang C, Wang JL, Zhou JC, Ma YY (2020) Extraction of power lines from laser point cloud based on residual clustering method. Acta Geodaetica et Cartographica Sinica. 49: 883-892.

23. Ma WF, Wang JL, Wang C, Xi XH, Wang P (2019) An Extraction Algorithm of Power Lines from Airborne LiDAR Point Clouds. Journal of Geomatics Science and Technology. 36: 39-44.

24. Ma ZY, Pang Y, Li ZY, Lu H, Liu LX, Chen BW (2019) Fine classification of near-ground point cloud based on terrestrial laser scanning and detection of forest fallen wood. J. Remote Sens. 23: 743-755.

25. Maltamo M, Eerikäinen K, Packalén P, Hyyppä J (2006) Estimation of stem volume using laser scanning-based canopy height metrics. Forestry: An International Journal of Forest Research, 79凶 217-229. https://doi.org/10.1093/forestry/cpl007

26. Meng XY (2006) Forest Mensuration(the 3nd dition). Chinese Forestry Publishing House, Beijing. pp. 10-17. 
27. Olofsson K, Holmgren J, Olsson H (2014) Tree Stem and Height Measurements using Terrestrial Laser Scanning and the RANSAC Algorithm. Remote Sens. 6: 4323-4344. https://doi.org/10.3390/rs6054323.

28. Pang Y, Li ZY, Chen BW, Liang XJ (2019) Status and Development of Spaceborne Lidar Applications in Forestry. Aerospace Shanghai. 36》 20-28. https://doi.org/10.19328/j.cnki.1006-1630.2019.03.003

29. Tao SL, Wu FF, Guo QH, Wang YC, Li WK, Xue BL, Hu XY, Li P, Tian D, Li C, Yao H, Li YM, Xu GC, Fang JY (2015) Segmenting tree crowns from terrestrial and mobile LiDAR data by exploring ecological theories. ISPRS-J. Photogramm. Remote Sens. 110: 66-76. https://doi.org/10.1016/j.isprsjprs.2015.10.007

30. Wang XY, Xing YQ, You HT, Xing T, Shu S (2019) TLS point cloud classification of forest based on nearby geometric features. Journal of Beijing Forestry University. 41: 138-146. https://doi.org/10.13332/j.1000-1522.20180308.

31. Wang Y (2019) 3D Trunk Surface Simulation of Chinese Fir Based on Terrestrial Laser Scanning Point Cloud Data; Central South University of Forestry \& Technology: Changsha, China.

32. Wang YJ, Li K, Lu LJ (2019) Power line classification from airborne LiDAR data via multi-scale neighborhood features. Bulletin of Surveying and Mapping. 04: 21-25. https://doi.org/10.13474/j.cnki.11-2246.2019.0106.

33. Xia SB, Wang C, Pan FF, Xi XH, Zeng HC, Liu H (2015) Detecting Stem in Dense and Homogeneous Forest Using Single-Scan TLS. Forests. 6: 3923-3945. https://doi.org/10.3390/f6113923.

34. Xie YY, Wang B, Yao Y, Yang L, Gao Y, Zhang ZM, Lin LX (2020) Quantification of vertical community structure of subtropical evergreen broad-leaved forest community using UAV-Lidar data. Acta Ecol. Sin. 40: 940-951. https://doi.org/10.5846/stxb201809282112.

35. Xuan W, Hua XH, Zou JG, He XX, Zhao BF (2019) A new method of normal estimation for point cloud based on adaptive optimal neighborhoods. Science of Surveying and Mapping. 44: 101-108+116. https://doi.org/10.16251/j.cnki.1009-2307.2019.10.015.

36. Yan YJ, Fan SH, Guan FY (2018) Research Progress in TLS Technology in Forest Investigation. World Forestry Research. 31: 42-47. https://doi.org/10.13348/j.cnki.sjlyyj.2018.0055.y.

37. Yang BS, Dong Z (2013) A shape-based segmentation method for mobile laser scanning point clouds. ISPRS-J. Photogramm. Remote Sens. 81: 19-30. https://doi.org/10.1016/j.isprsjprs.2013.04.002.

38. Yu XW, Liang X, Hyyppae J, Kankare V, Vastaranta M, Holopainen M (2013) Stem biomass estimation based on stem reconstruction from terrestrial laser scanning point clouds. Remote Sens. Lett. 4: 344-353. https://doi.org/10.1080/2150704X.2012.734931

39. Yu YH, Wang JL, Liu GJ, Cheng F (2019) Forest Leaf Area Index Inversion Based on Landsat OLI Data in the Shangri-La City. J. Indian Soc. Remote Sens. 47: 967-976. https://doi.org/10.1007/s12524-019-00950-6.

40. Zhao HJ, Tan JX, Lei J, Shu ZX (2020) Transmission line extraction from UAV-LiDAR based on geometric features. Bulletin of Surveying and Mapping. S1: 145-150. 
https://doi.org/10.13474/j.cnki.11-2246.2020.0535.

41. Zhou RQ, Xu ZH, Peng CG, Zhang F, Jiang WS (2019) A JointBoost-based classification method of high voltage transmission corridor from airborne LiDAR point cloud. Science of Surveying and Mapping. 44: 21-27+33. https://doi.org/10.16251/j.cnki.1009-2307.2019.03.004.

\section{Figures}

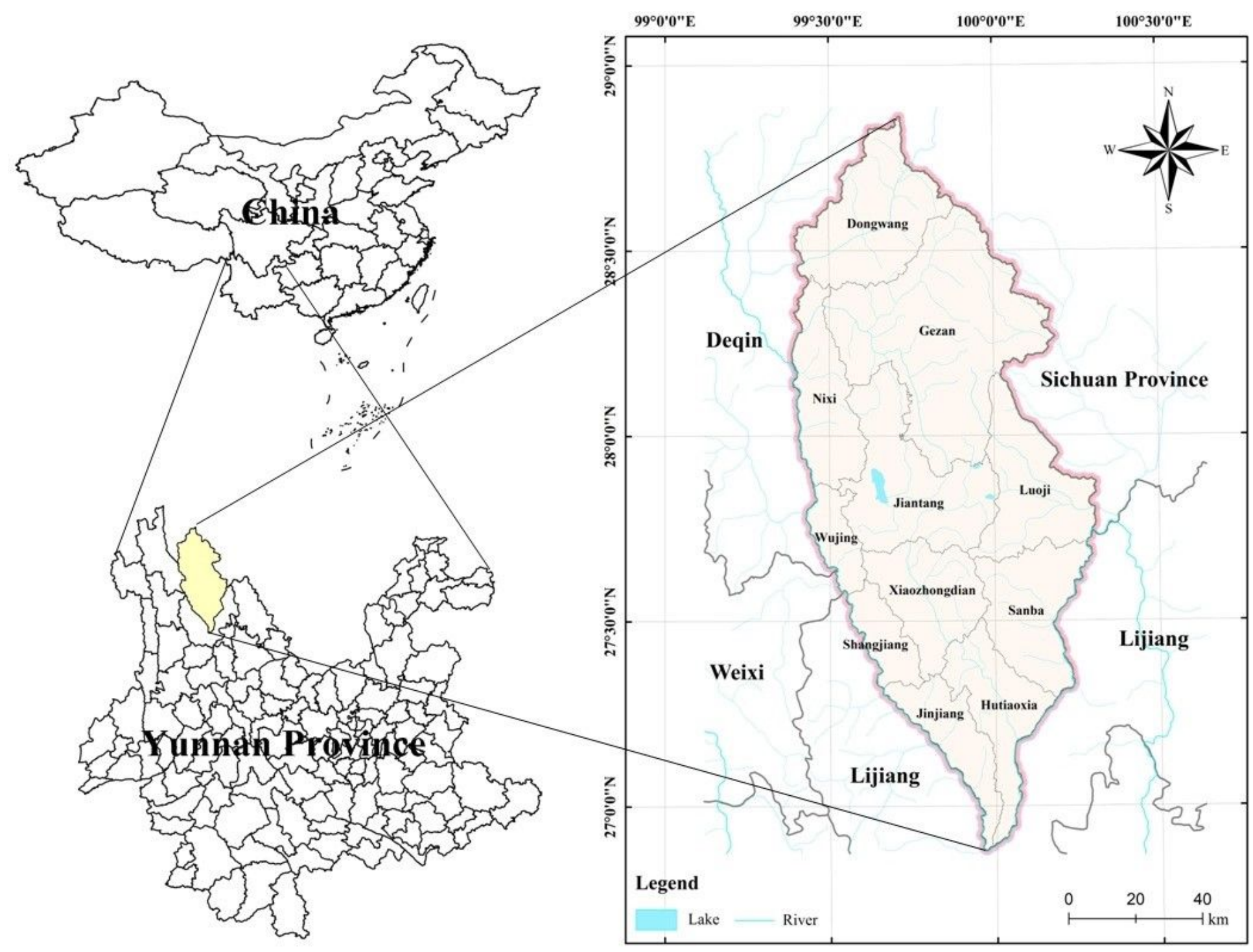

Figure 1

Study area Note: The designations employed and the presentation of the material on this map do not imply the expression of any opinion whatsoever on the part of Research Square concerning the legal status of any country, territory, city or area or of its authorities, or concerning the delimitation of its frontiers or boundaries. This map has been provided by the authors. 


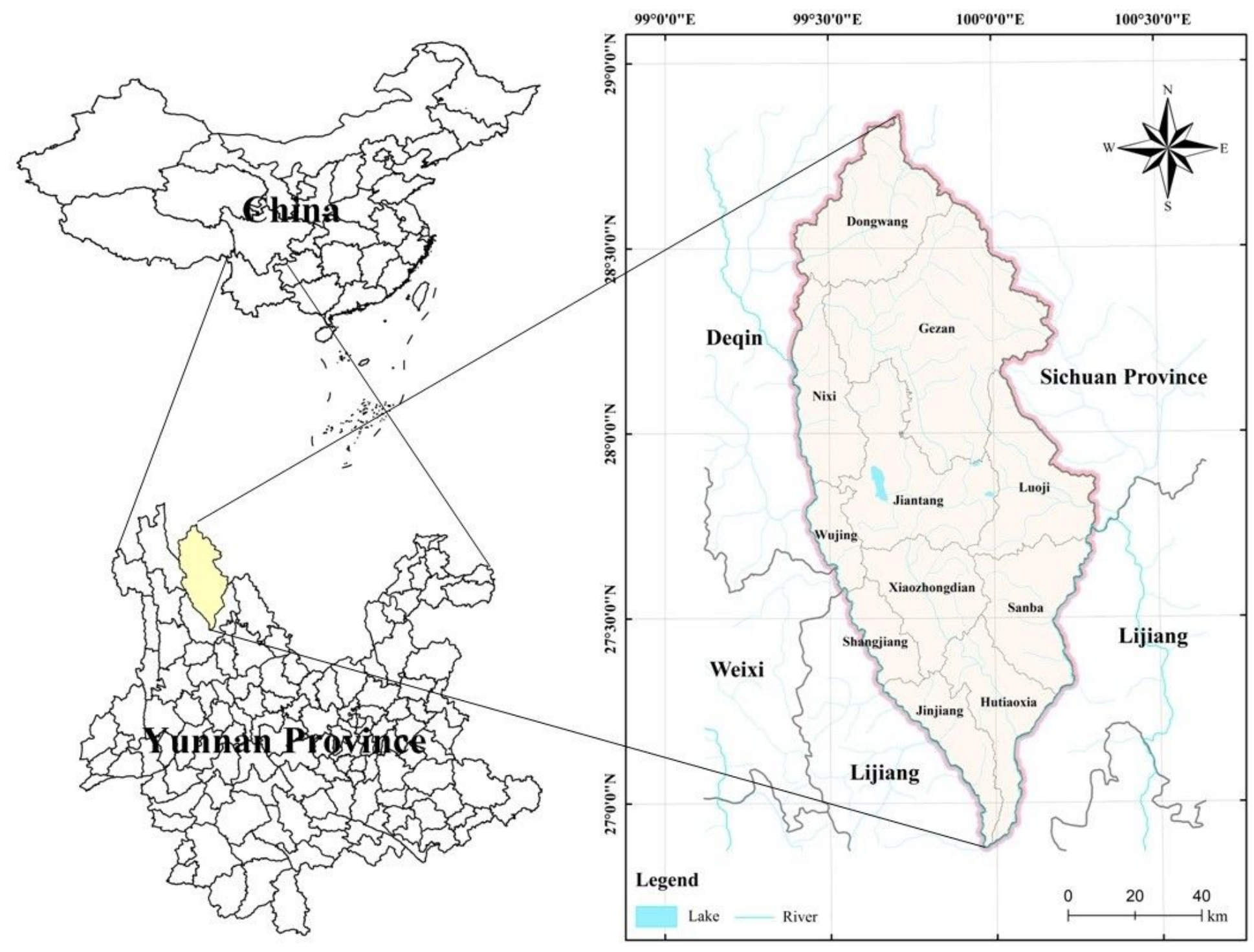

Figure 1

Study area Note: The designations employed and the presentation of the material on this map do not imply the expression of any opinion whatsoever on the part of Research Square concerning the legal status of any country, territory, city or area or of its authorities, or concerning the delimitation of its frontiers or boundaries. This map has been provided by the authors. 


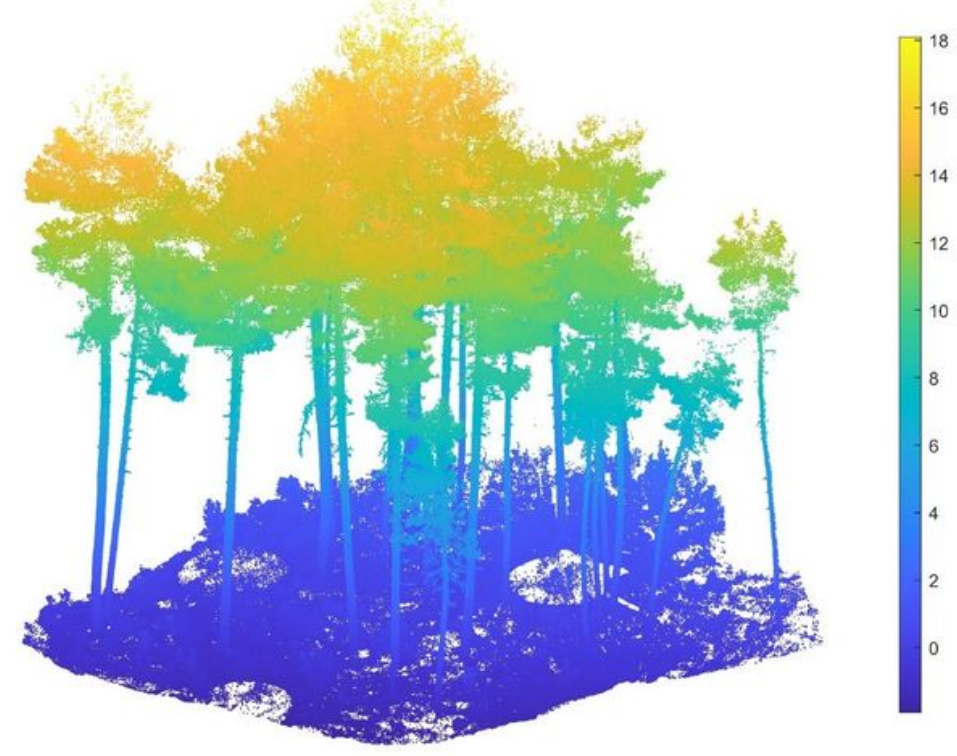

(a)

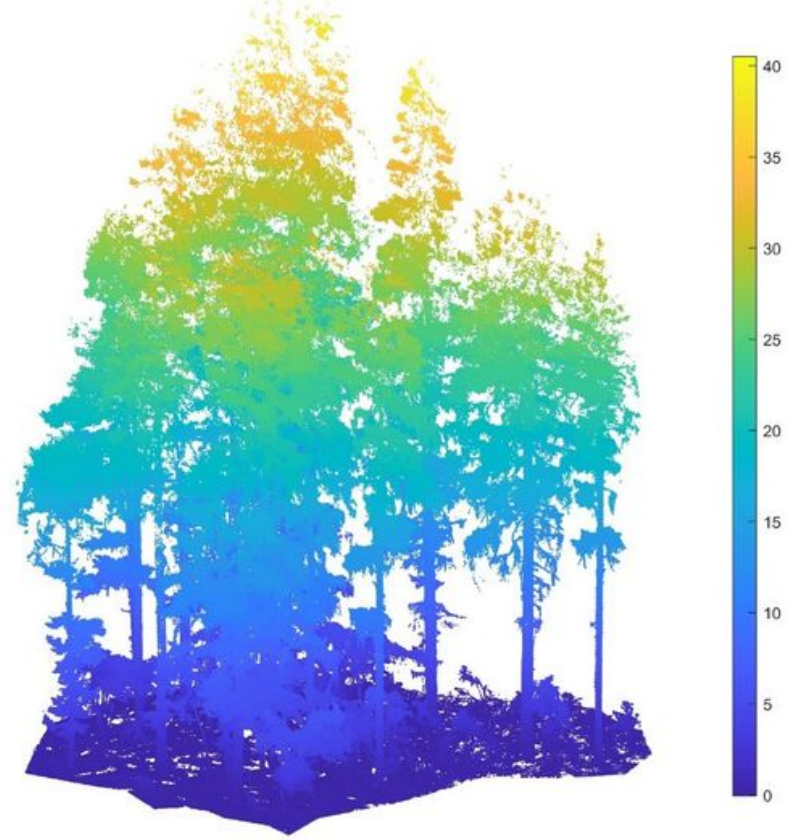

(b)

Figure 2

Experimental point cloud data. (a) Pinus densata Mast.; (b) Picea asperata Mast.

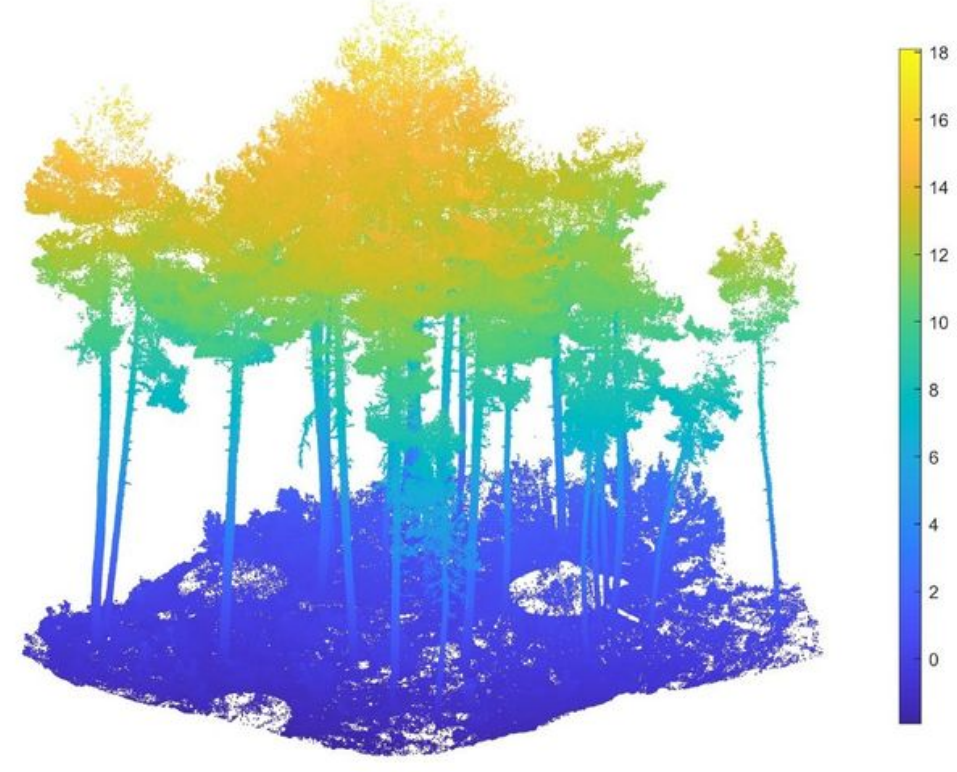

(a)

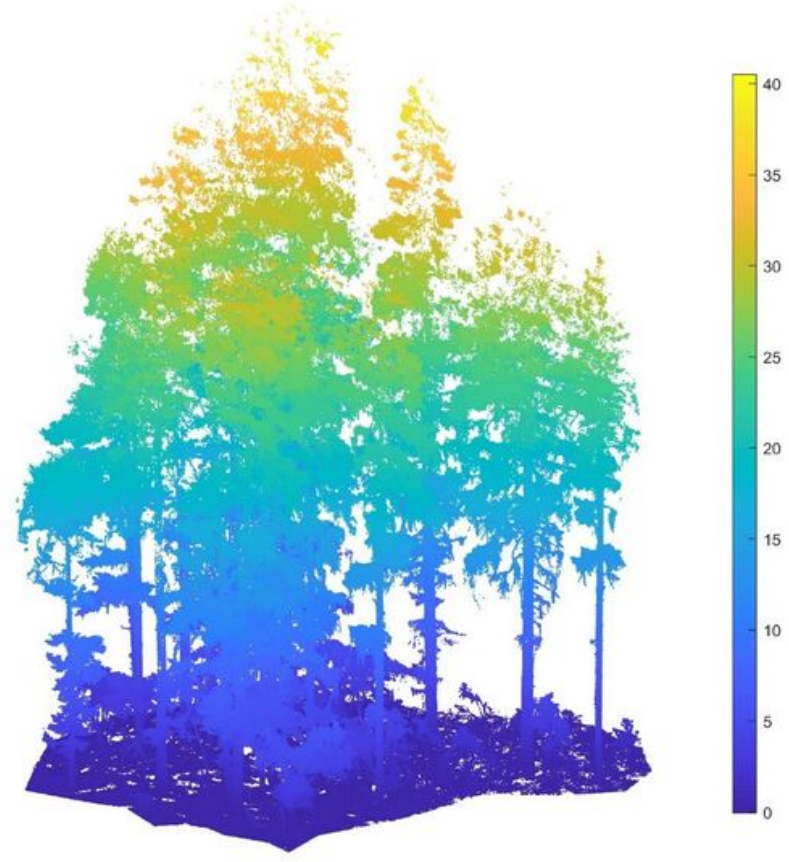

(b)

\section{Figure 2}

Experimental point cloud data. (a) Pinus densata Mast.; (b) Picea asperata Mast. 

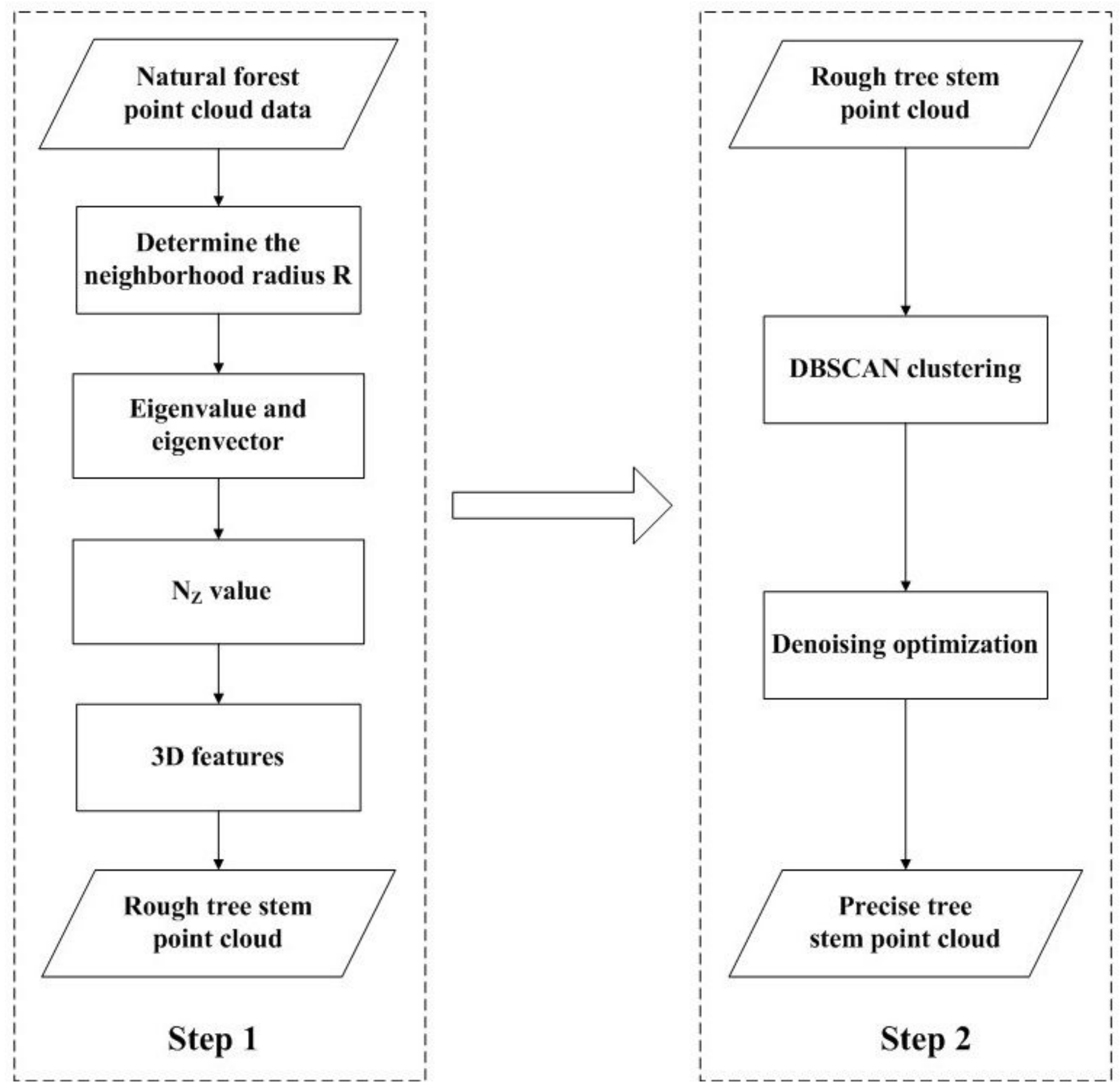

Figure 3

Technology roadmap 

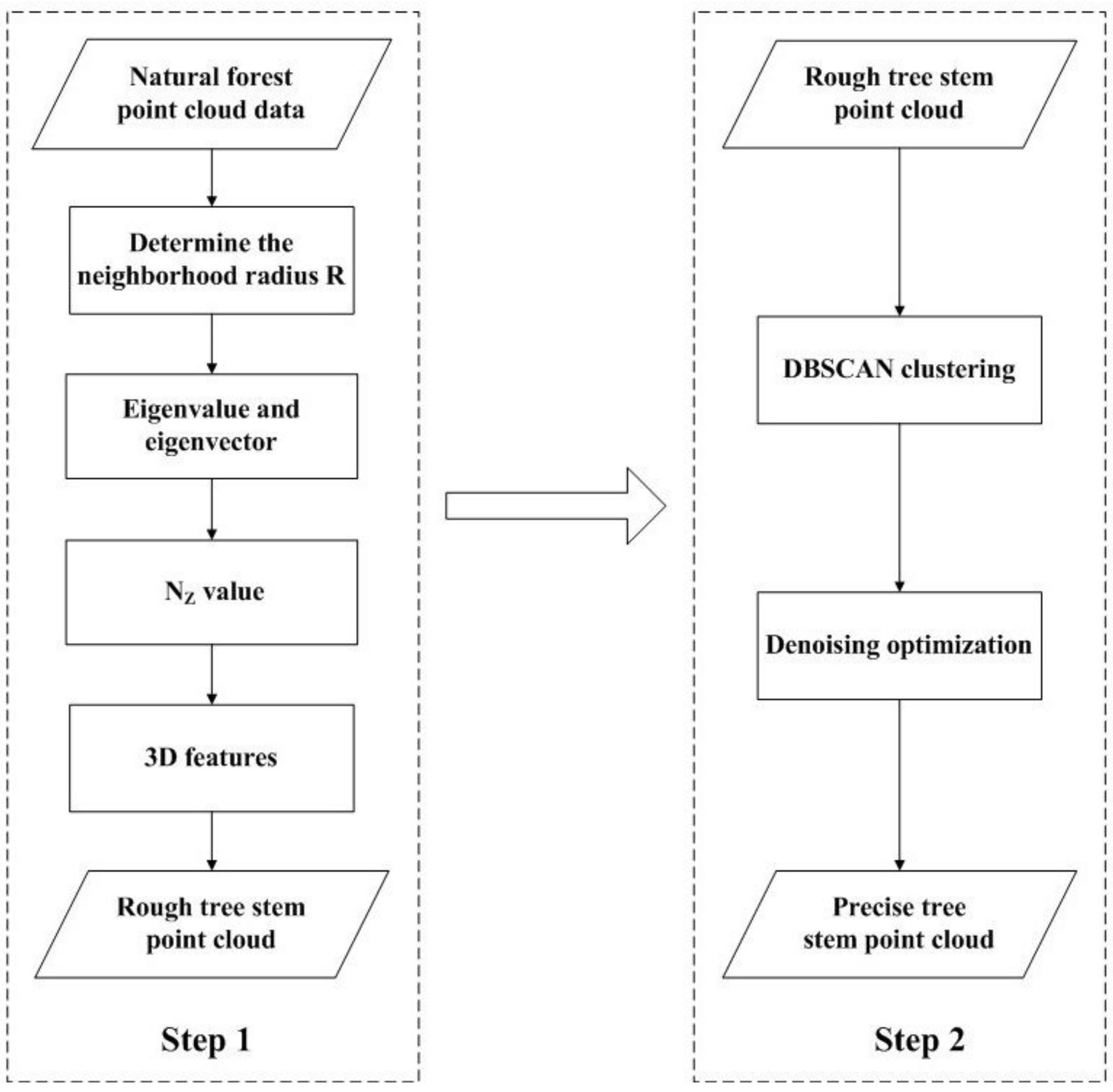

Figure 3

Technology roadmap 


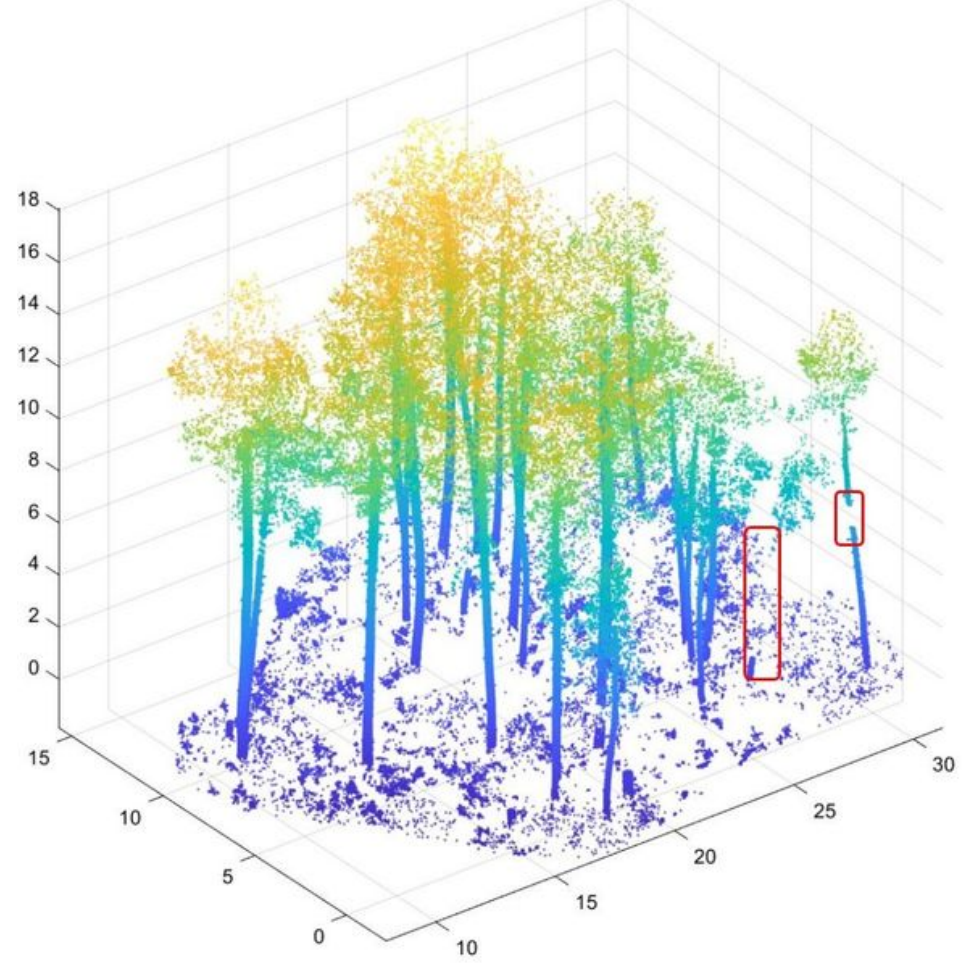

(a)

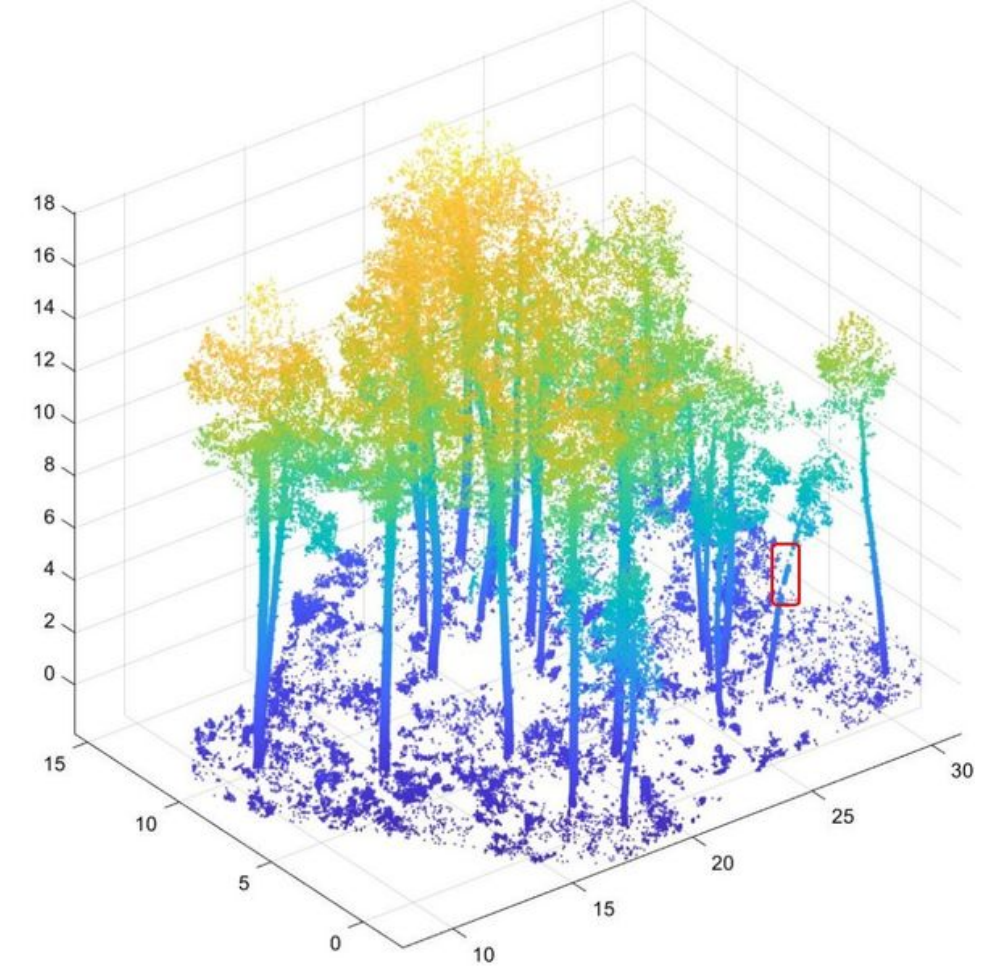

(b)

\section{Figure 4}

Filter out results for non-stem points. (a) $\mathrm{T}=0.1 ;$ (b) $\mathrm{T}=0.2$

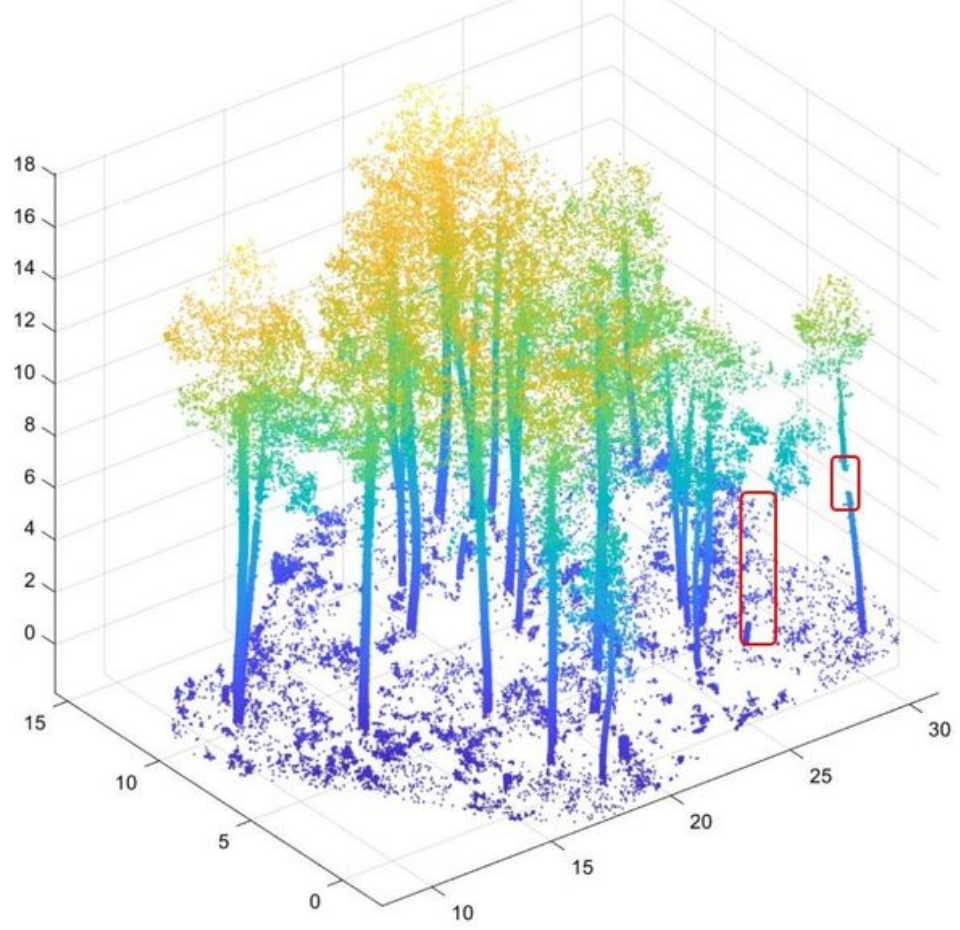

(a)

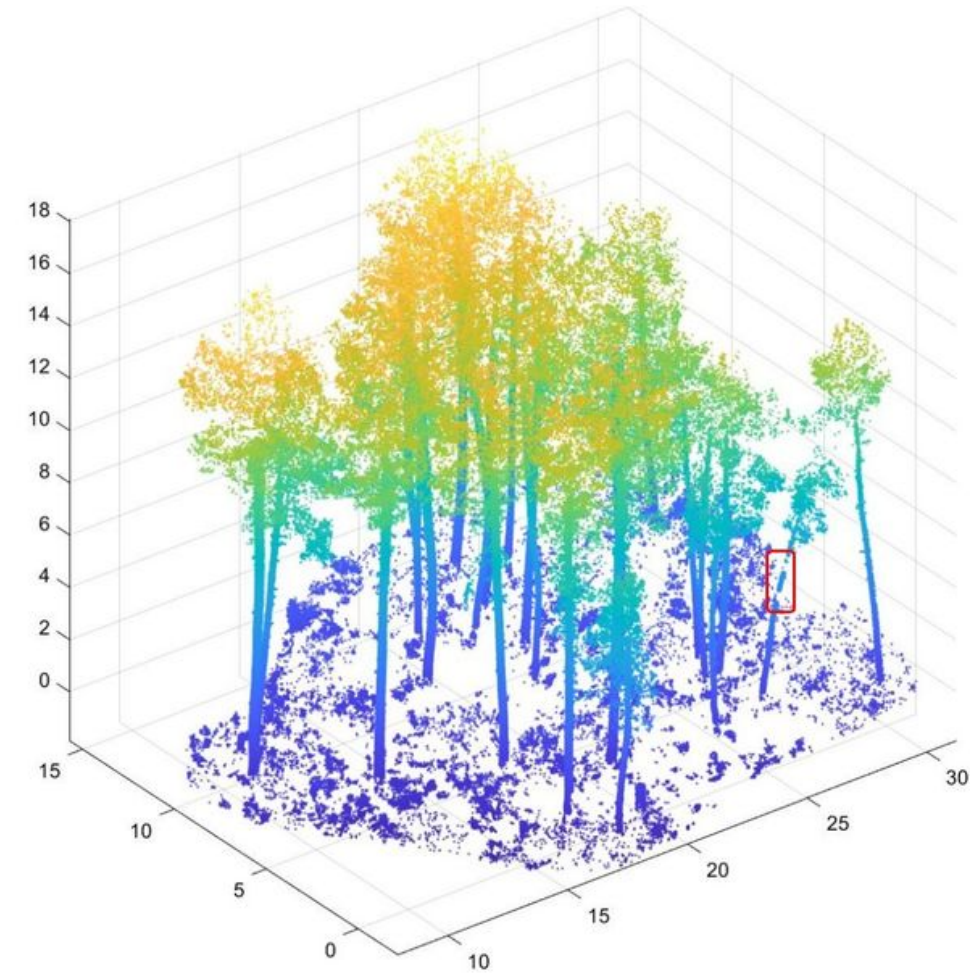

(b)

Figure 4 
Filter out results for non-stem points. (a) $\mathrm{T}=0.1 ;(b) \mathrm{T}=0.2$

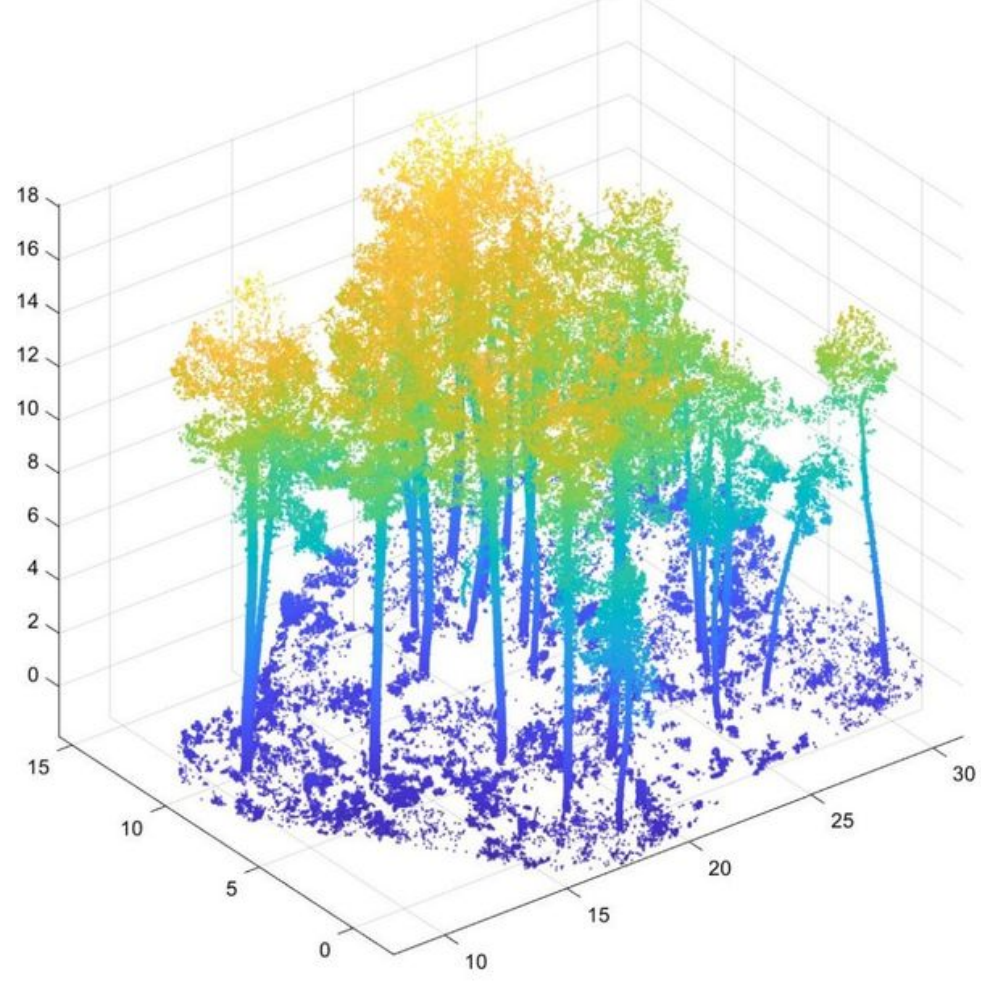

(a)

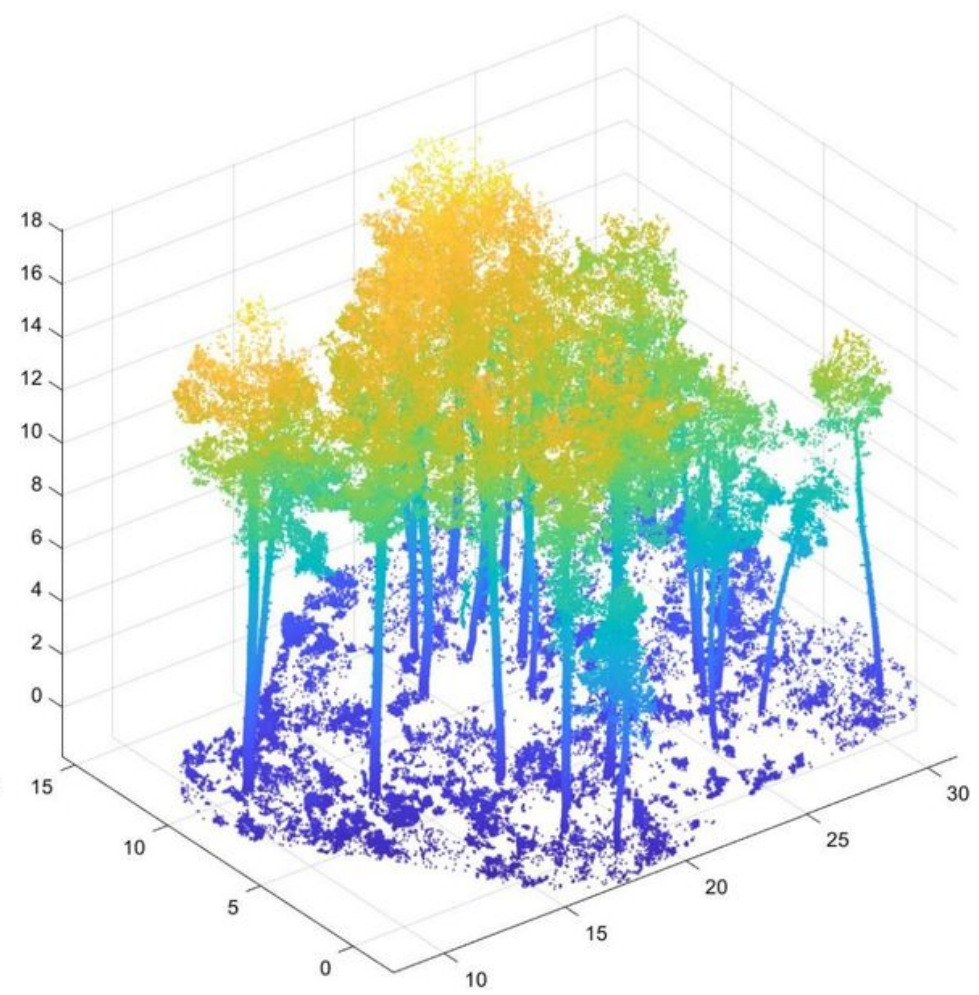

(b)

Figure 5

Filter out results for non-stem points. $(a) T=0.3 ;(b) T=0.4$ 


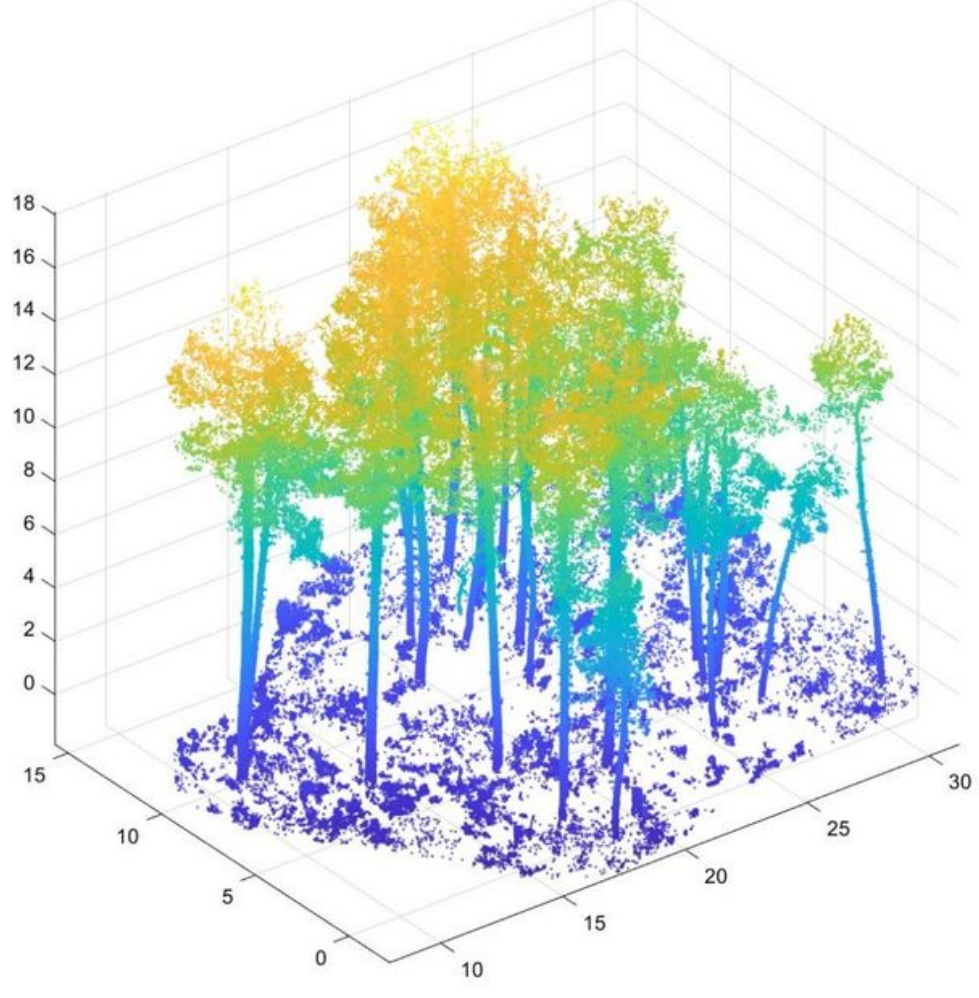

(a)

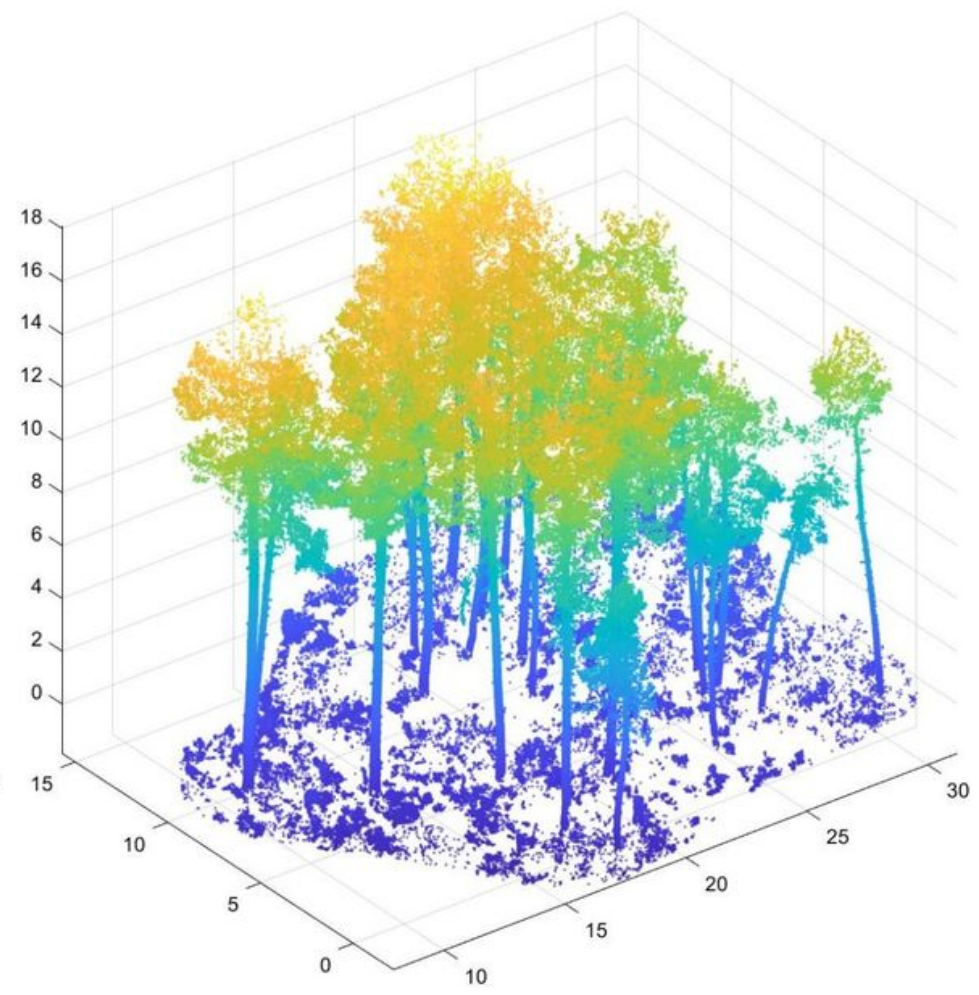

(b)

\section{Figure 5}

Filter out results for non-stem points. (a) $\mathrm{T}=0.3 ;(b) \mathrm{T}=0.4$

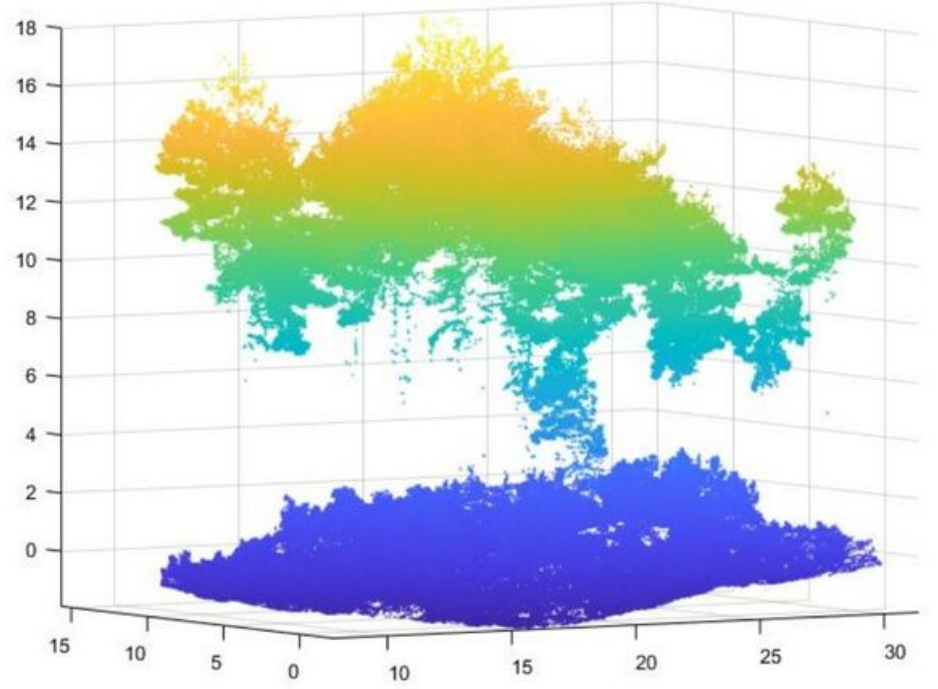

(a)

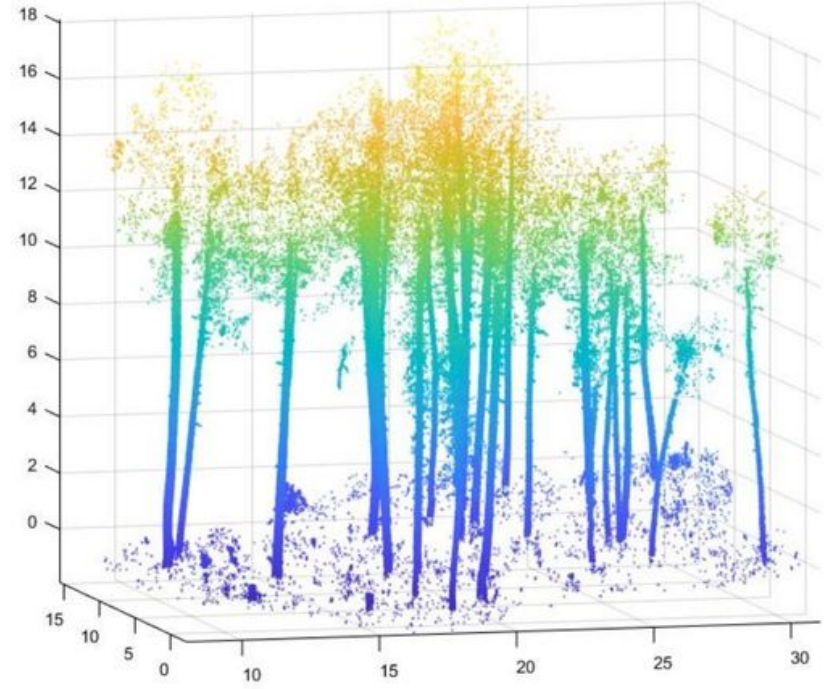

(b)

\section{Figure 6}

The result of 5 looped with $T=0.3$. (a) Non-stem point cloud; (b) Stem point cloud 


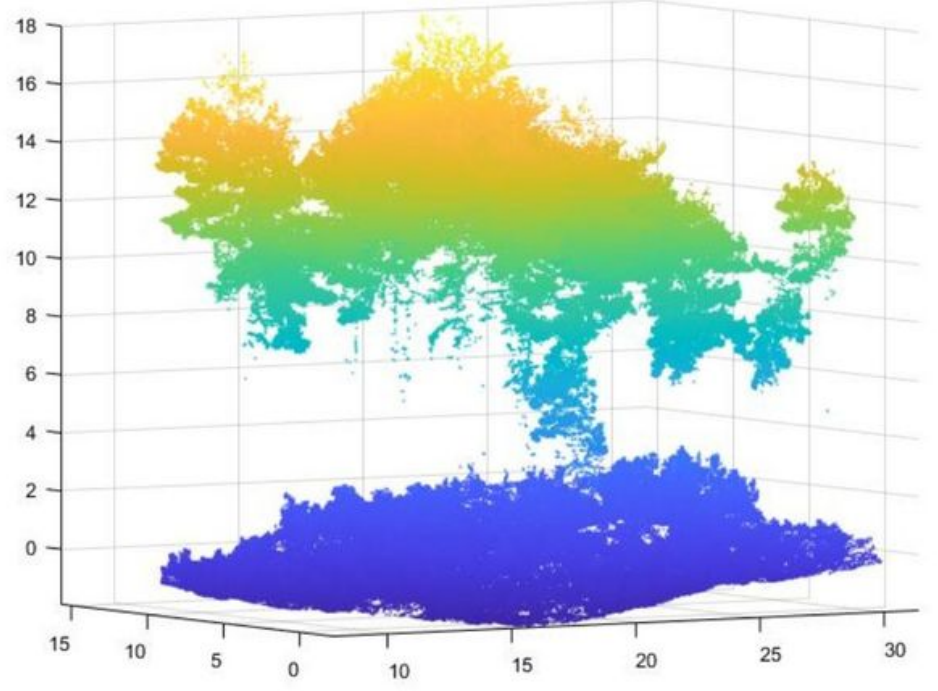

(a)

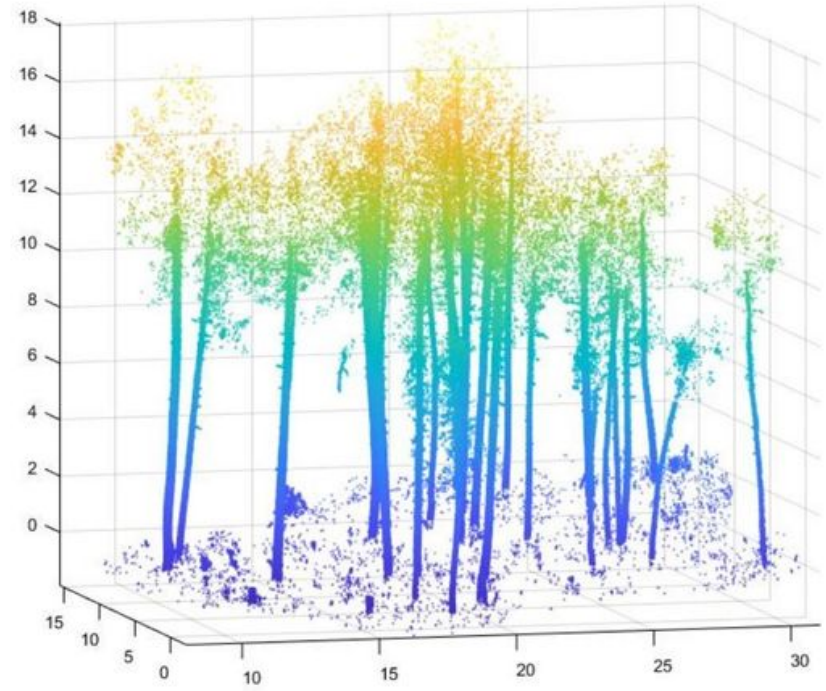

(b)

Figure 6

The result of 5 looped with $T=0.3$. (a) Non-stem point cloud; (b) Stem point cloud

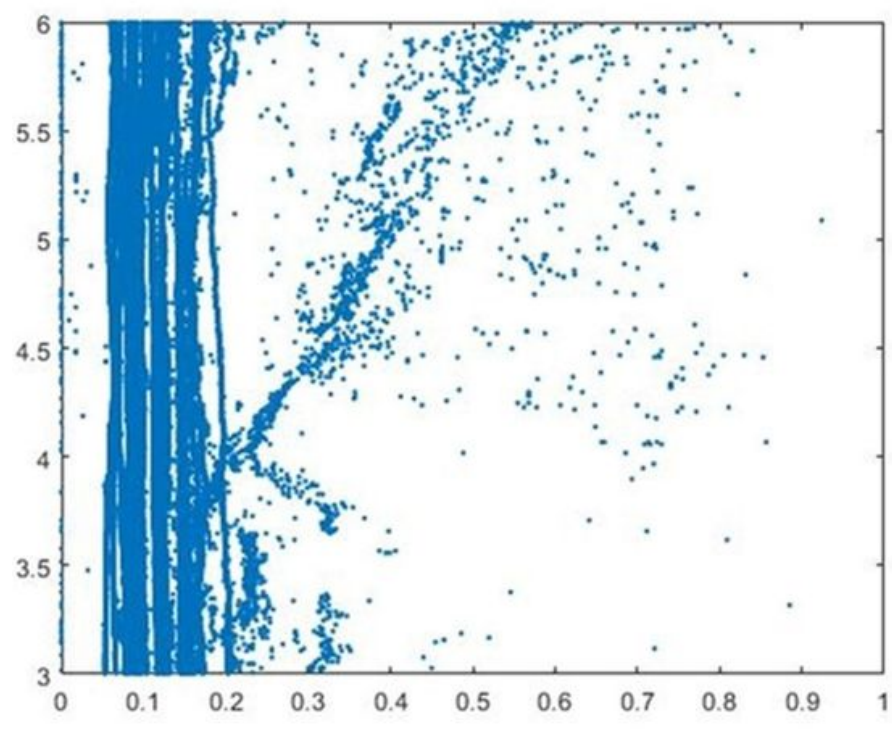

(a)

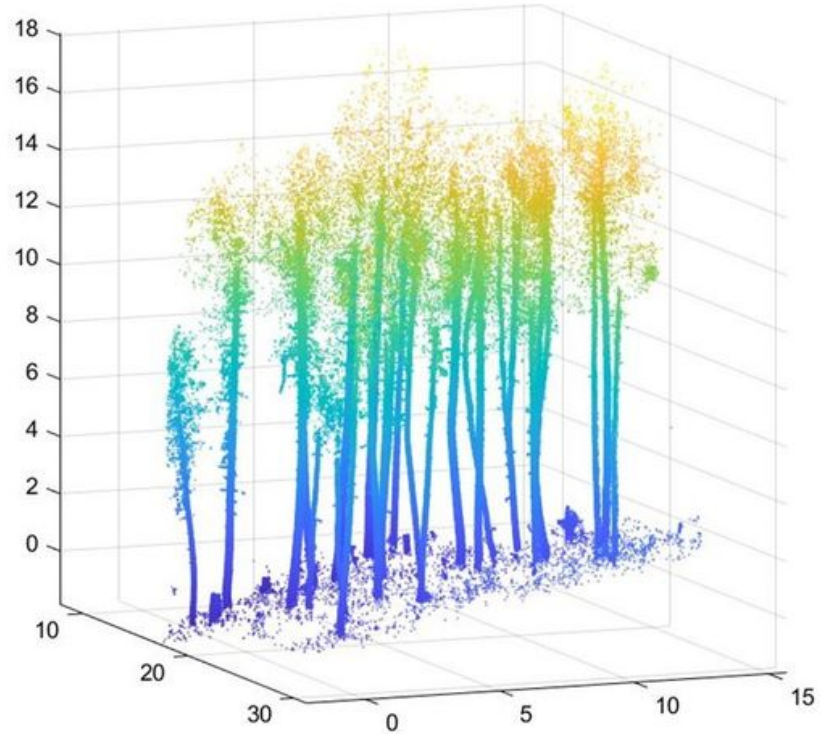

(b)

Figure 7

The result of rough stem extraction. (a) 3D value distribution of 3m-6m tree stem; (b) Rough stem extraction results 


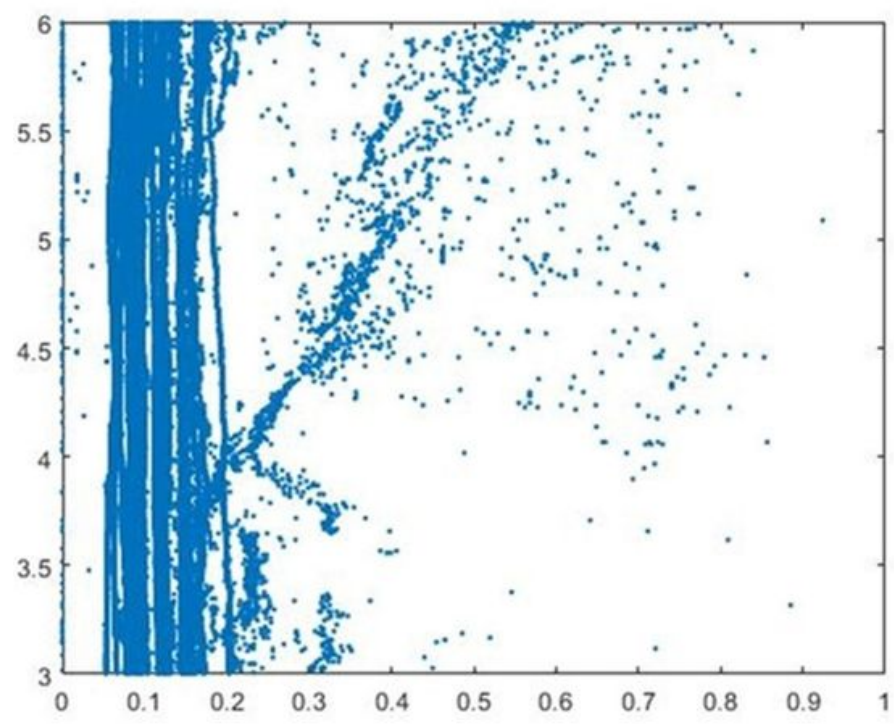

(a)

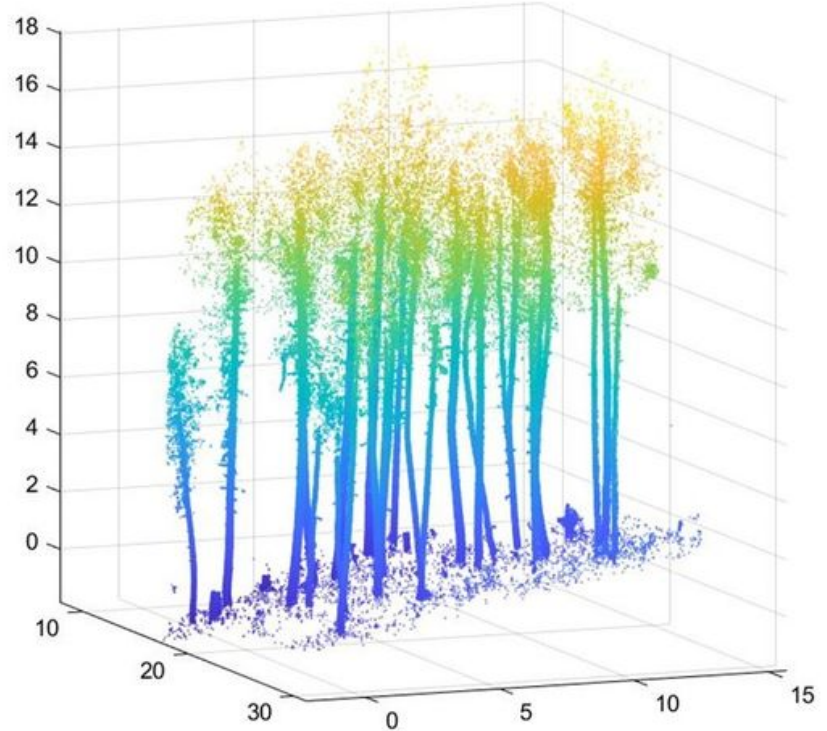

(b)

Figure 7

The result of rough stem extraction. (a) 3D value distribution of 3m-6m tree stem; (b) Rough stem extraction results

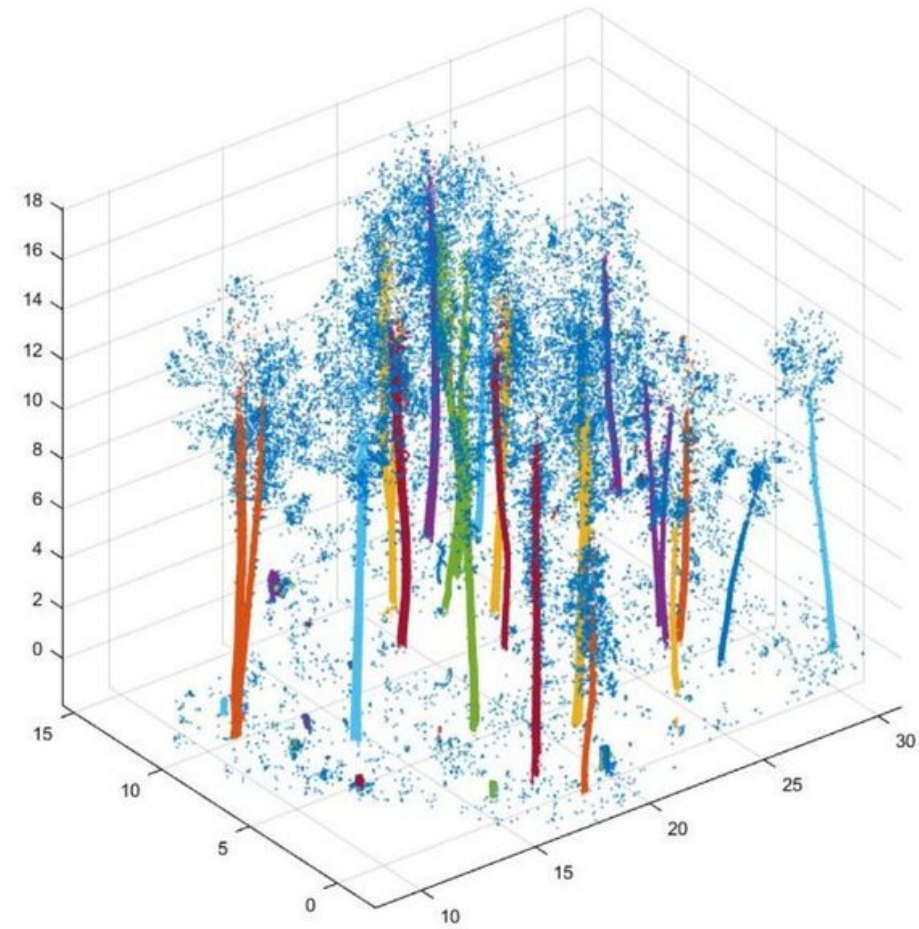

(a)

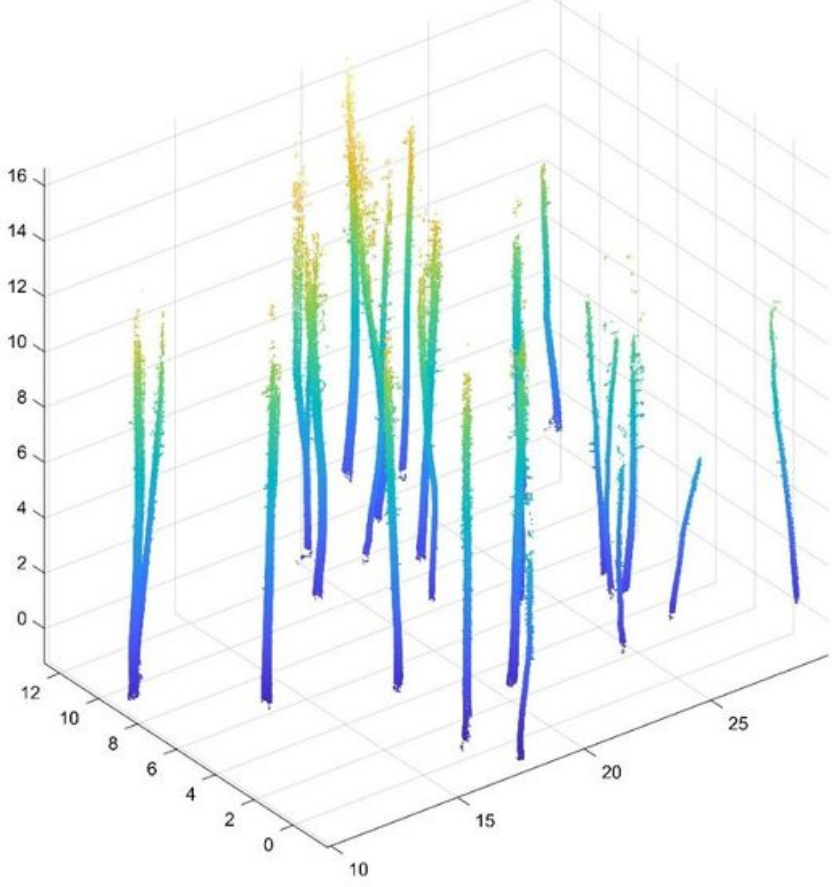

(b)

Figure 8

The precise stem extraction result of Pinus densata Mast. sample plot. (a) DBSCAN tree stem clustering results; (b) Precise stem extraction results 


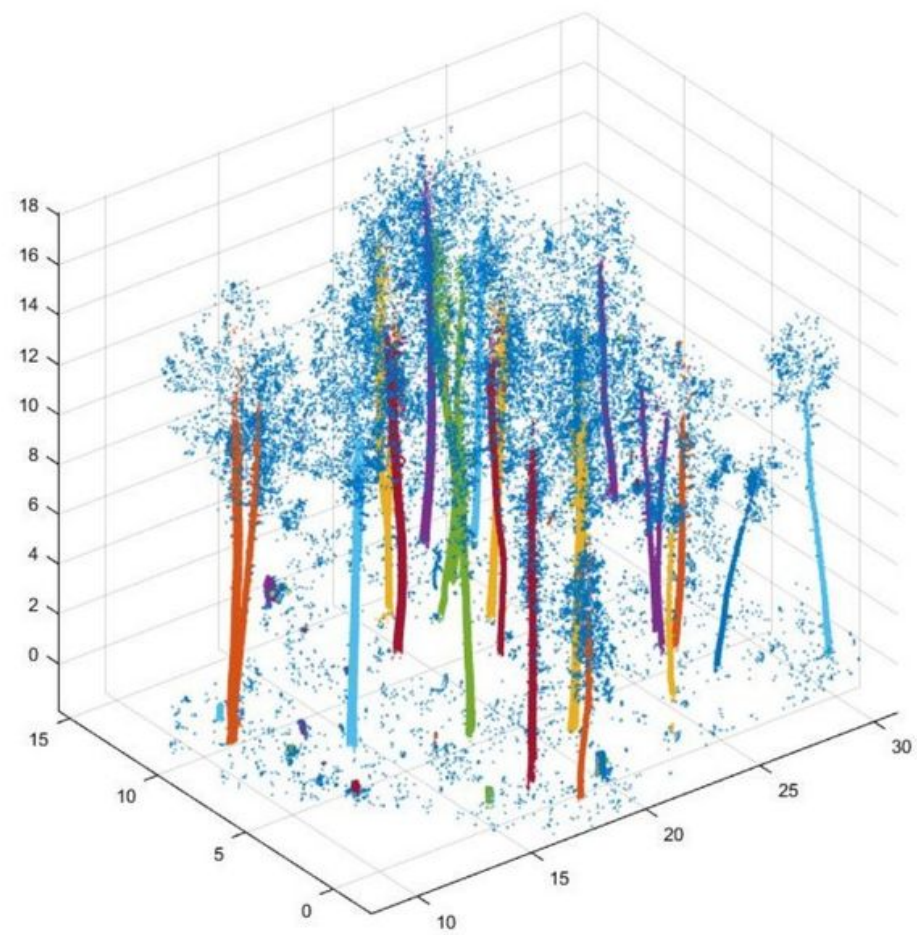

(a)

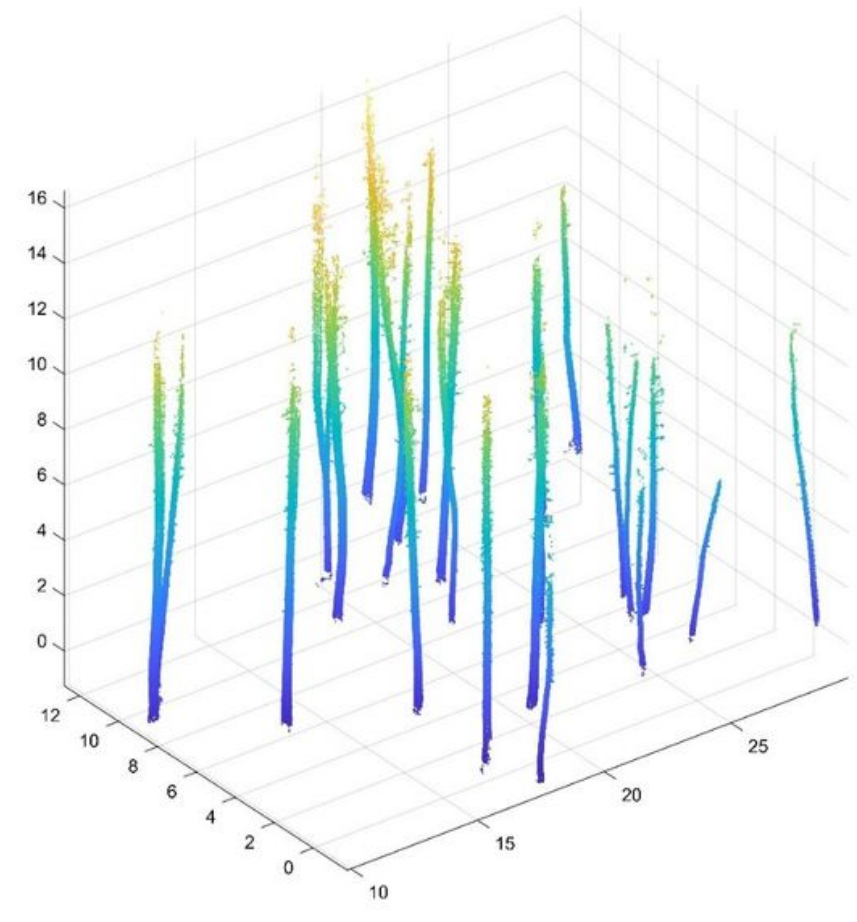

(b)

Figure 8

The precise stem extraction result of Pinus densata Mast. sample plot. (a) DBSCAN tree stem clustering results; (b) Precise stem extraction results 


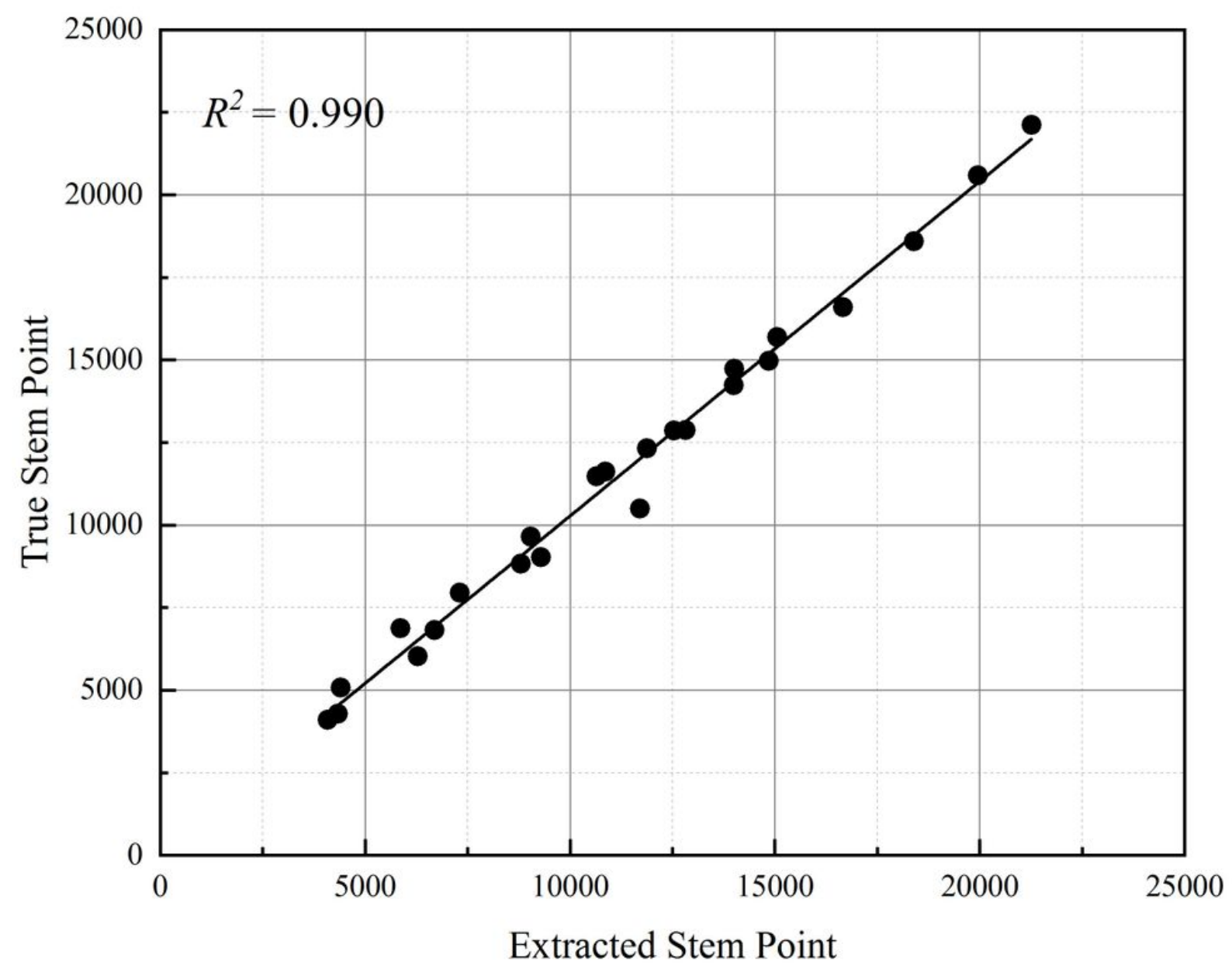

Figure 9

The tree stem extraction results accuracy analysis of Pinus densata Mast. sample plot 


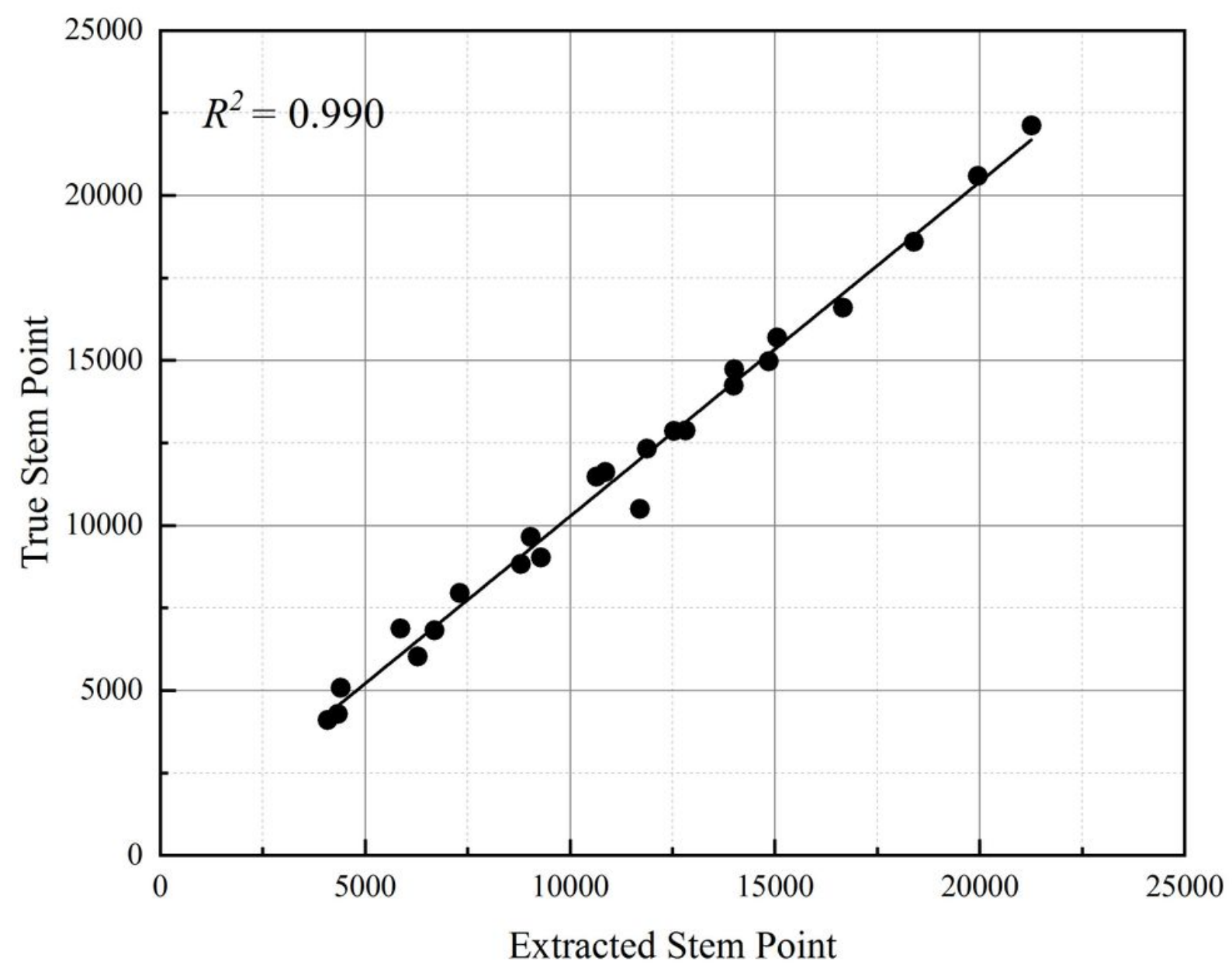

Figure 9

The tree stem extraction results accuracy analysis of Pinus densata Mast. sample plot 


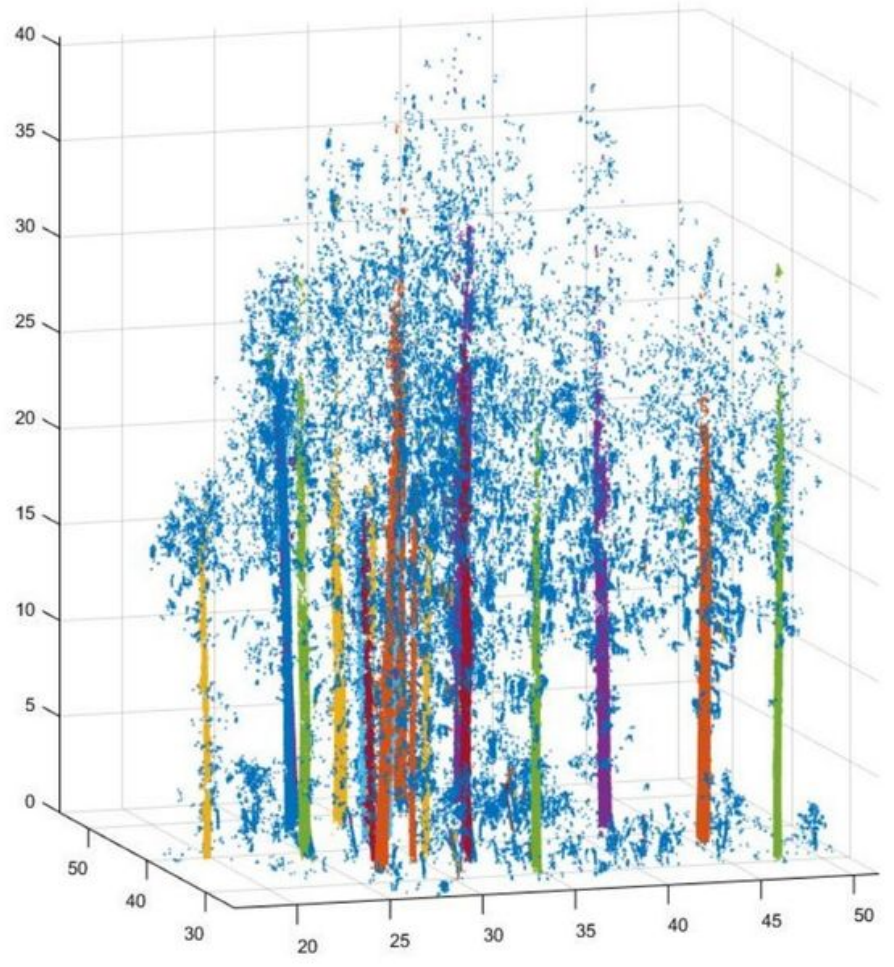

(a)

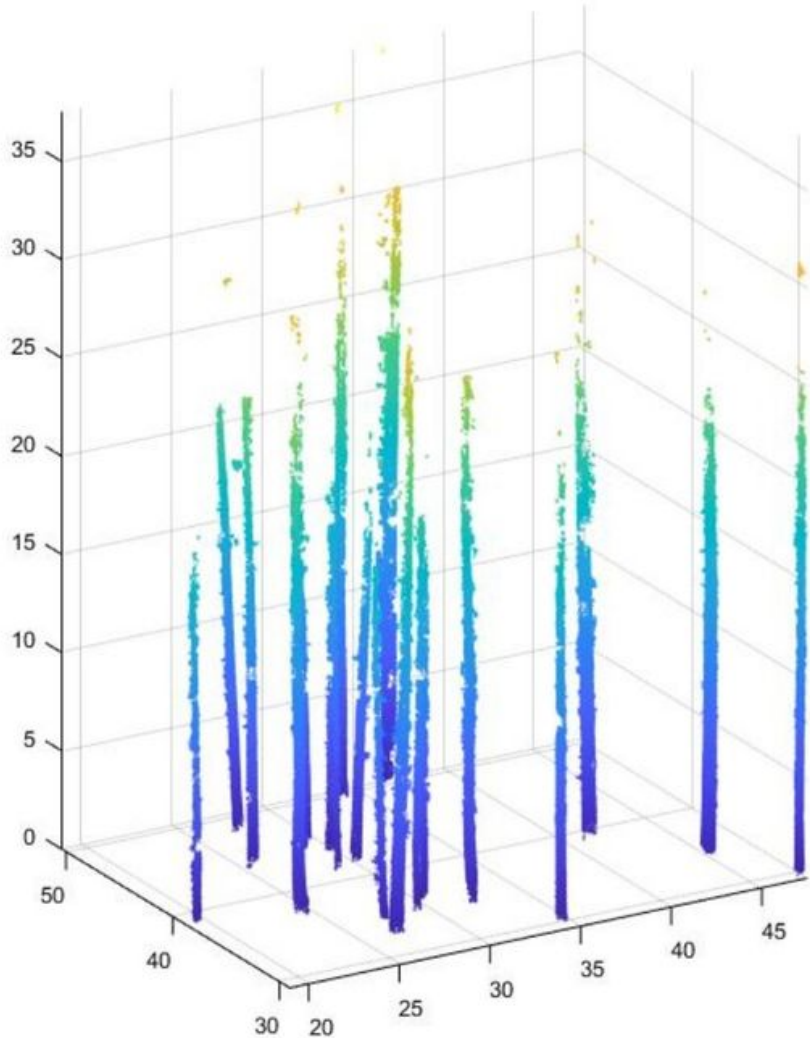

(b)

Figure 10

The precise stem extraction result of Picea asperata Mast. sample plot. (a) DBSCAN tree stem clustering results; (b) Precise stem extraction results 


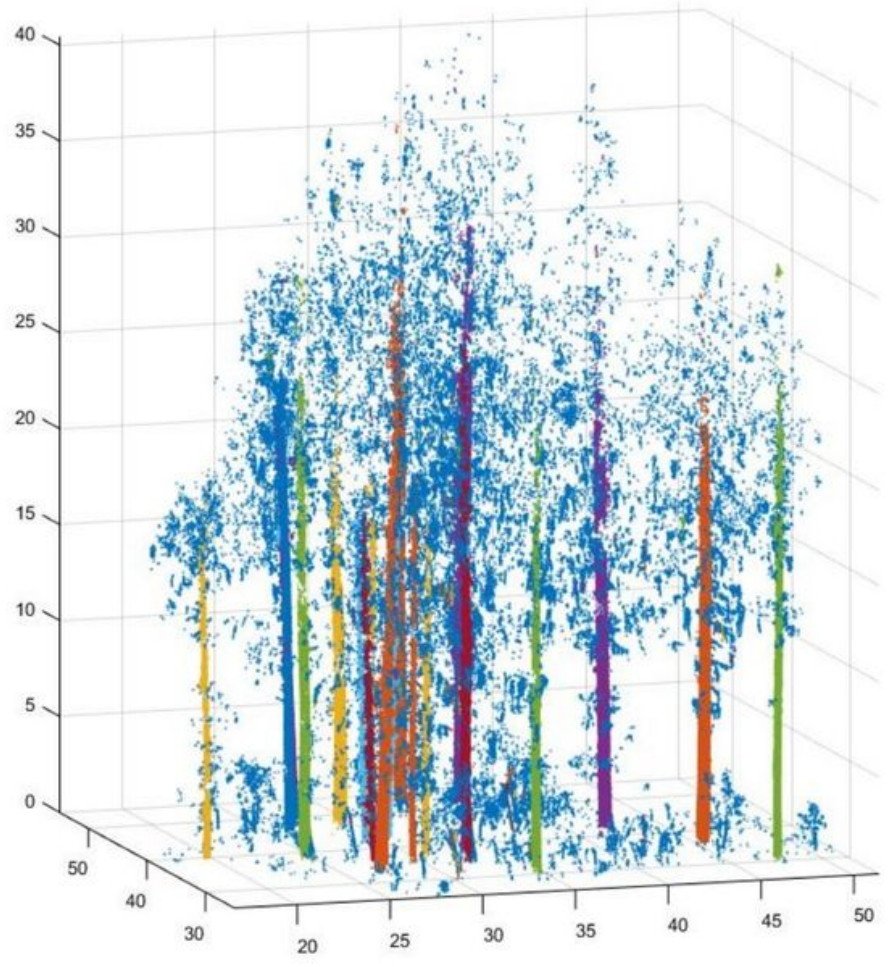

(a)

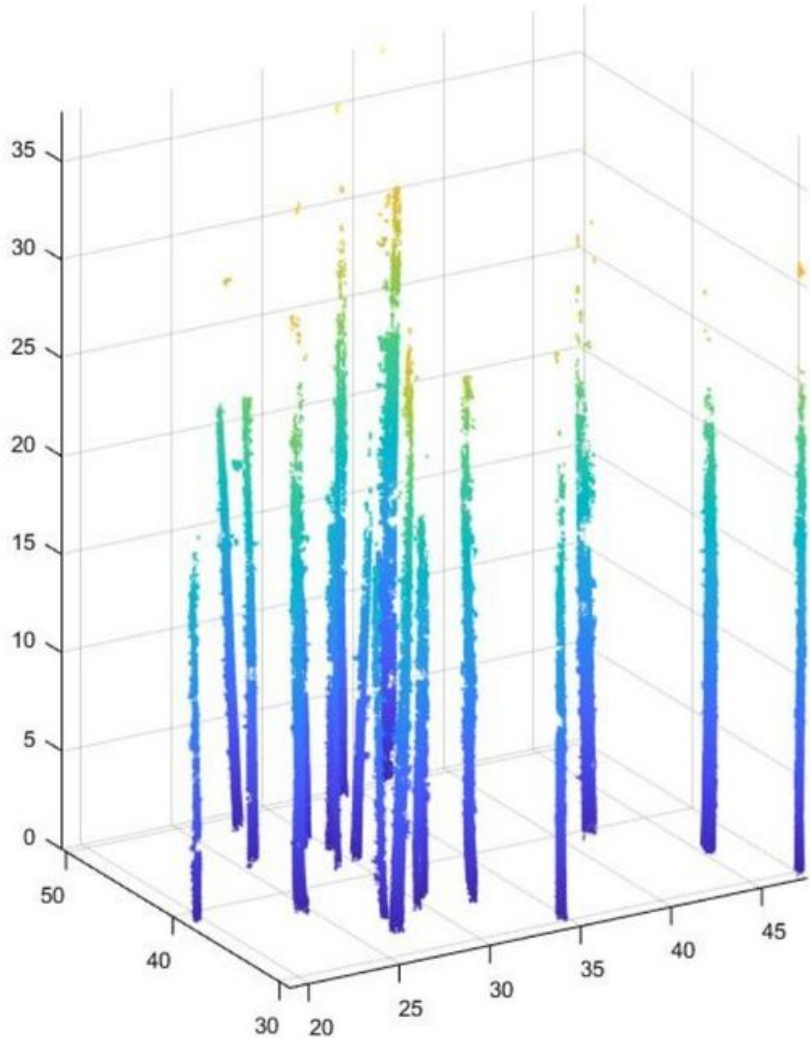

(b)

Figure 10

The precise stem extraction result of Picea asperata Mast. sample plot. (a) DBSCAN tree stem clustering results; (b) Precise stem extraction results 


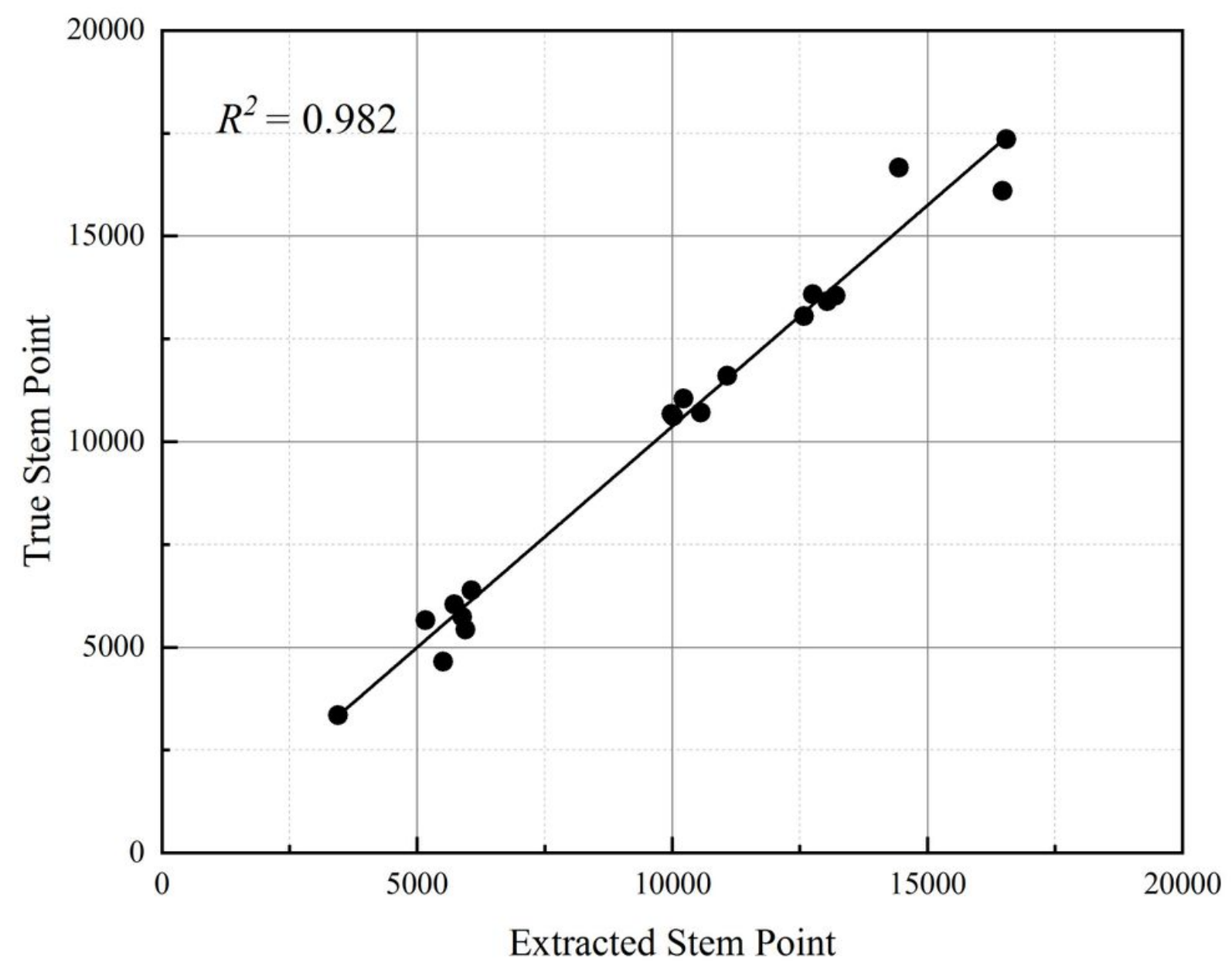

Figure 11

The tree stem extraction results accuracy analysis of Picea asperata Mast. sample plot 


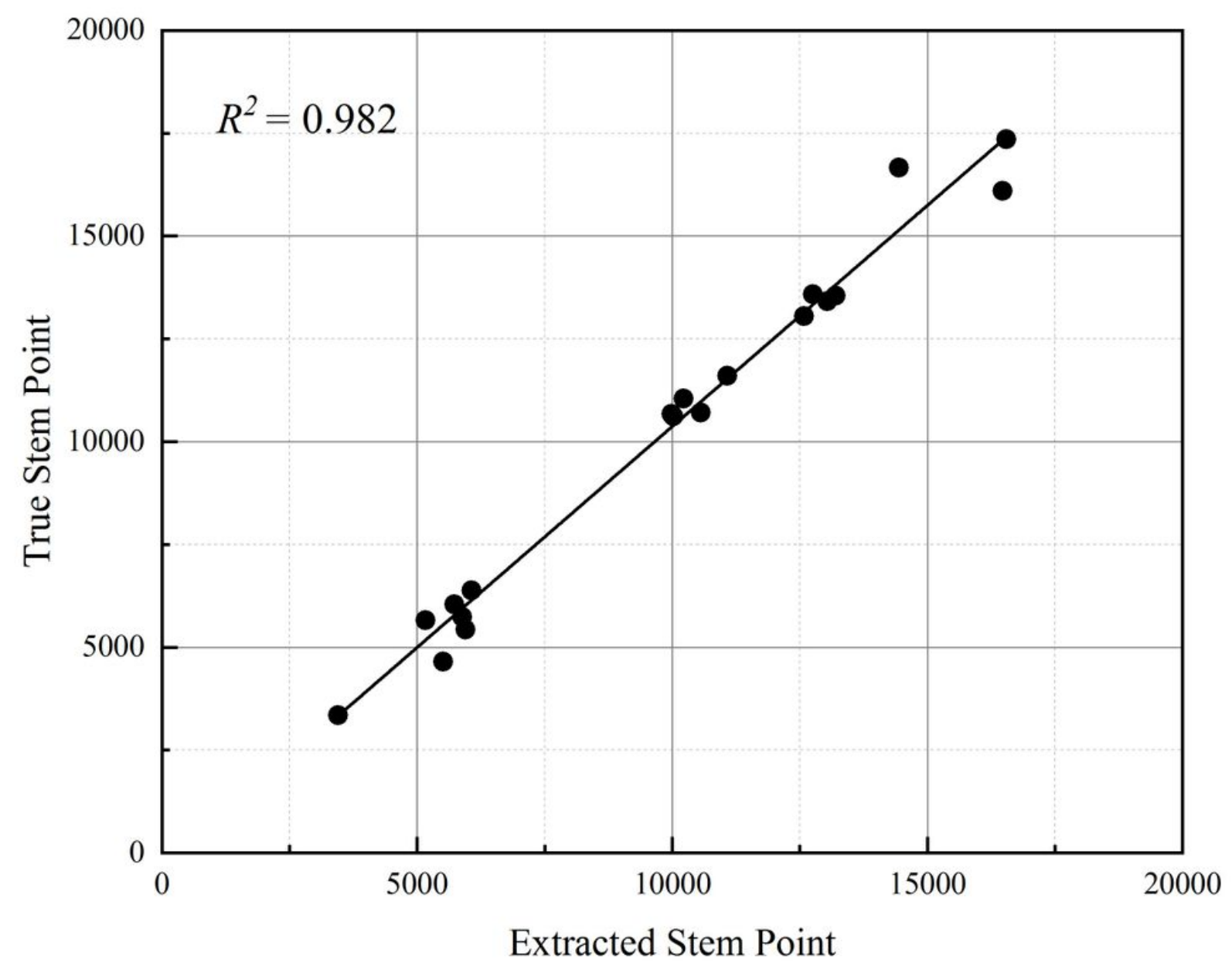

Figure 11

The tree stem extraction results accuracy analysis of Picea asperata Mast. sample plot 

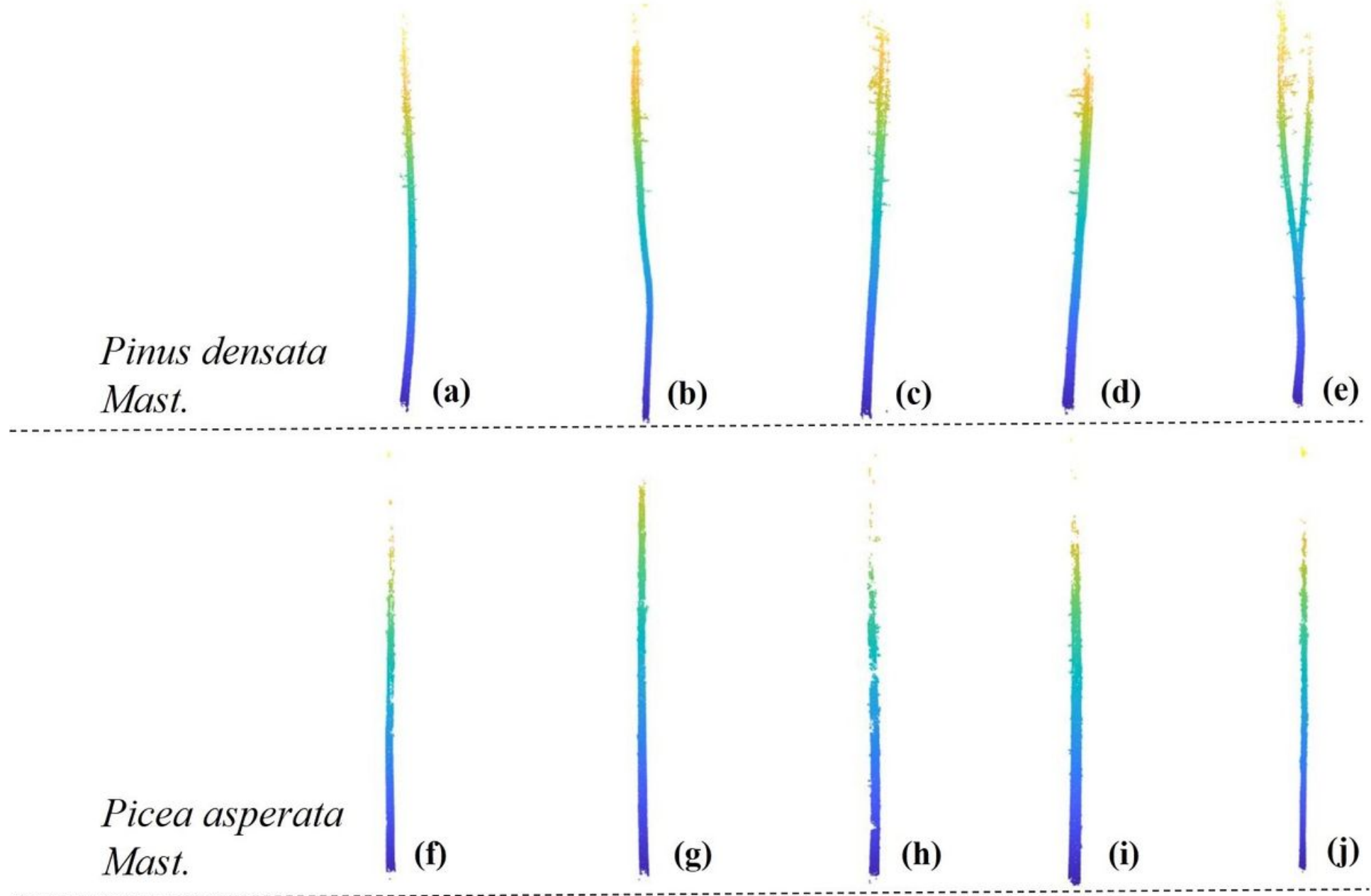

Figure 12

Stem comparative analysis of two sample plots 

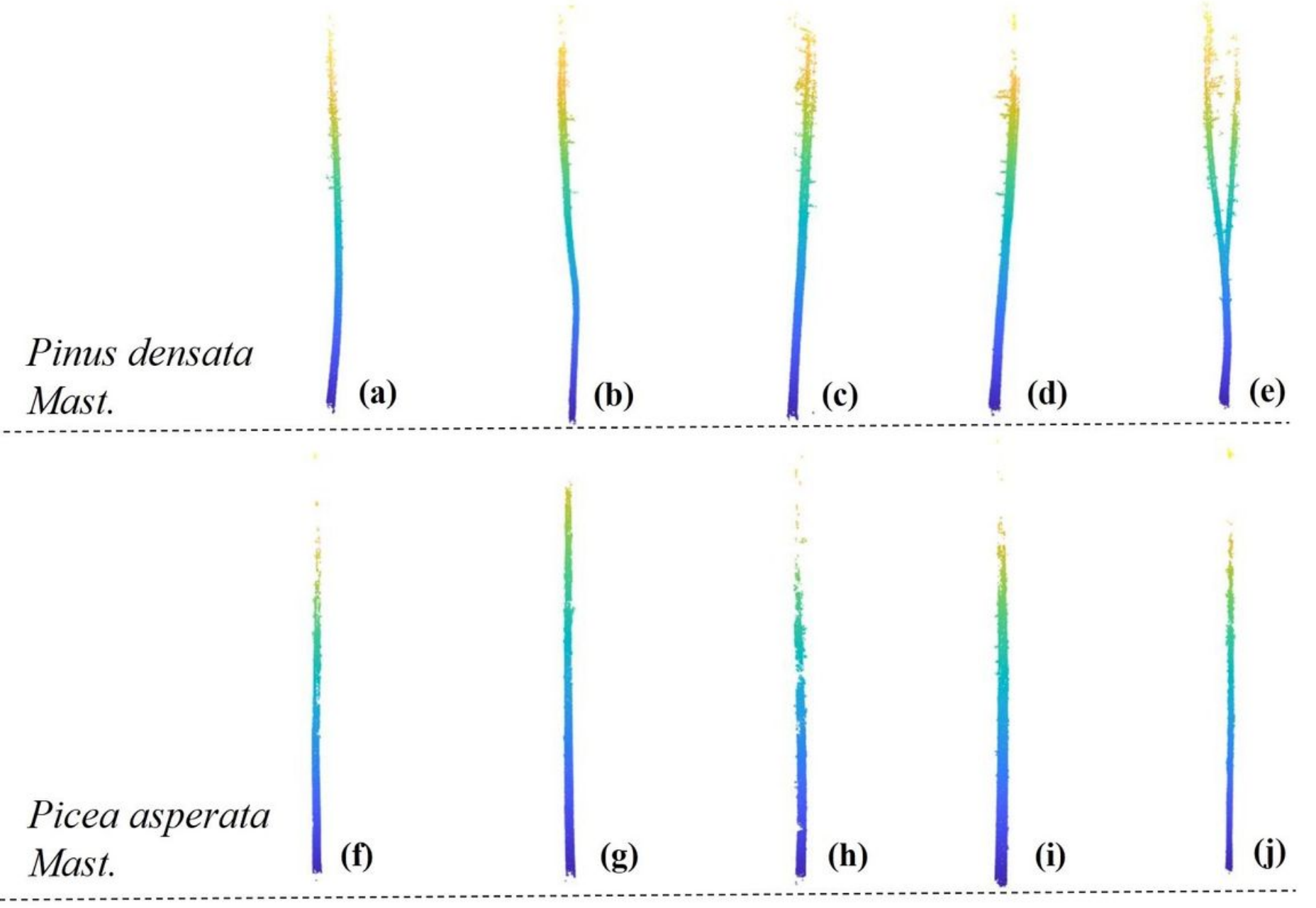

Figure 12

Stem comparative analysis of two sample plots 